\title{
ALGORITHMS FOR STRONGLY STABLE IDEALS
}

\author{
DENNIS MOORE AND UWE NAGEL
}

\begin{abstract}
Strongly stable monomial ideals are important in algebraic geometry, commutative algebra, and combinatorics. Prompted, for example, by combinatorial approaches for studying Hilbert schemes and the existence of maximal total Betti numbers among saturated ideals with a given Hilbert polynomial, in this paper we present three algorithms to produce all strongly stable ideals with certain prescribed properties: the saturated strongly stable ideals with a given Hilbert polynomial, the almost lexsegment ideals with a given Hilbert polynomial, and the saturated strongly stable ideals with a given Hilbert function. We also establish results for estimating the complexity of our algorithms.
\end{abstract}

\section{INTRODUCTION}

Strongly stable monomial ideals arise naturally in algebraic geometry, commutative algebra, and combinatorics. In fact, Galligo, Bayer and Stillman showed that the generic initial ideal of a homogeneous ideal is Borel-fixed. In characteristic zero, Borel-fixed ideals are strongly stable (see, e.g., [6] or [17]). Shifting is a combinatorial technique that studies a given simplicial complex by modifying the given complex to a simpler one while preserving essential properties. Strongly stable ideals figure prominently in the algebraic approach to shifting (see, e.g., 17]). A Hilbert scheme parametrizes the closed subschemes of a projective space with a fixed Hilbert polynomial. Its scheme structure is very complex. Strongly stable ideals are the basis for combinatorial approaches for studying Hilbert schemes (see, e.g., [14, 24, 26], 22]).

Building on work by Reeves 24] and Gehrs 9, in this paper we present an algorithm that produces all saturated strongly stable ideals of a polynomial ring with a given Hilbert polynomial. We restrict ourselves to saturated ideals for two reasons. With respect to the reverse lexicographic order, the generic initial ideal of an ideal is saturated if and only if the ideal is saturated, and the homogeneous ideal of a closed subscheme is saturated. Moreover, the number of strongly stable ideals with a given Hilbert polynomial is not finite.

We also develop two related algorithms. Recently, Caviglia and Murai (see [4]) established that in the set of all saturated homogeneous ideals of a polynomial ring with a given Hilbert polynomial there exists an ideal whose total Betti numbers are

Received by the editor October 12, 2011 and, in revised form, January 9, 2013.

2010 Mathematics Subject Classification. Primary 14Q20, 13P99.

Key words and phrases. Hilbert polynomial, strongly stable ideal, Betti numbers, CastelnuovoMumford regularity, lexsegment ideal.

This work was partially supported by a grant from the Simons Foundation (\#208869 to Uwe Nagel).

The authors were also partially supported by the National Security Agency under Grant Number H98230-09-1-0032.

(C)2014 American Mathematical Society 
at least as large as the total Betti numbers of all other ideals in this set. This generalizes a result of Valla (see [27]) about ideals with constant Hilbert polynomial. Thanks to a result by Bigatti, Hulett, and Pardue, there must be an ideal with maximal Betti numbers that is saturated, strongly stable, and a lexsegment ideal when considered in a polynomial ring in one less variable. We call such an ideal almost lexsegment (see Definition 5.1). We show that a modification of our first algorithm produces all almost lexsegment ideals to a given Hilbert polynomial by computing only almost lexsegment ideals at every step. The algorithm reveals in particular that, for a given Hilbert polynomial, there can be many almost lexsegment ideals that achieve the maximal Betti numbers.

Our third algorithm produces all saturated strongly stable ideals with a given Hilbert function. They form a subset of the ideals obtained by the first algorithm. However, we present a more direct and more efficient algorithm for computing them. This algorithm can also be used to produce all (not necessarily saturated) strongly stable ideals with a given Hilbert series (see Remark 6.4).

This paper is organized as follows. In Section 2, we briefly recall some well-known properties of strongly stable ideals. For unexplained terminology and background, we refer to [6], [17, and [15].

In Section 3 we introduce certain algorithmic operations - called contractions and expansions of monomials - on the set of minimal generators of strongly stable ideals. These operations were first proposed in [24] and also considered in [9]. For greater efficiency, we use suitable modifications of these operations, and we describe their effect on the Hilbert polynomial.

The theoretical core for our algorithms is provided by Theorem 4.4. It states that all saturated strongly stable ideals with the same Hilbert polynomial can be computed by using expansions of minimal monomial generators. The proof of this result is constructive and leads to a new algorithm for finding all saturated ideals having a prescribed Hilbert polynomial (see Algorithm4.6). It also includes a sharp estimate on the number of steps the algorithm needs to generate a strongly stable ideal starting from a trivial ideal.

Algorithm 4.6 is modified in Section 5 in order to produce all almost lexsegment ideals to a given Hilbert polynomial (see Algorithm 5.8). These ideals represent all the Hilbert functions of saturated homogeneous ideals with the given Hilbert polynomial. We also present an algorithm for directly generating all saturated strongly stable ideals with a fixed Hilbert function (see Algorithm 6.1).

In Section 7 we discuss consequences of the complexity estimate in Theorem 4.4. In particular, we show that the number of saturated strongly stable ideals in a polynomial ring in $n$ variables with a given Hilbert polynomial $p$ depends only on $p$ and not on $n$, once $n$ is sufficiently large (see Proposition 7.3). Fixing the Hilbert polynomial, we also describe the ideals with the worst CastelnuovoMumford regularity (see Theorem 7.5 ).

We implemented all algorithms presented in this paper in the computer algebra system Macaulay2 [11. The files can be downloaded at the following address: http://www. ms. uky.edu/ dmoore/M2. 


\section{Strongly Stable ideals And some Properties}

Throughout this paper we denote by $R:=K\left[x_{0}, \ldots, x_{n}\right]$ the polynomial ring over an arbitrary field $K$. Also, we denote by $R^{(1)}:=K\left[x_{0}, \ldots, x_{n-1}\right]$ the polynomial ring where the last variable has been removed, and, more generally, $R^{(j)}:=$ $K\left[x_{0}, \ldots, x_{n-j}\right]$ is the polynomial ring where the last $j$ variables have been removed. We use multi-index notation: If $A=\left(a_{0}, \ldots, a_{n}\right)$ is an $n$-tuple of nonnegative integers we set $x^{A}=x_{0}^{a_{0}} \cdots x_{n}^{a_{n}}$. Moreover, if $x^{A} \neq 1$, the max index of $x^{A}$ is

$$
\max \left(x^{A}\right):=\max \left\{i: a_{i}>0\right\}=\max \left\{i: x_{i} \mid x^{A}\right\} .
$$

Definition 2.1. A monomial ideal $I \subset R$ is called a strongly stable ideal if, for every monomial $x^{A} \in I$ and $x_{j} \mid x^{A}$, we have $\frac{x_{i}}{x_{j}} \cdot x^{A} \in I$ whenever $0 \leq i<j$.

Remark and Definition 2.2. Let $I \subset R$ be a strongly stable ideal.

(i) To determine whether an ideal is strongly stable, it is sufficient to check that the minimal monomial generators of the ideal satisfy the criterion in Definition 2.1.

(ii) The saturation of $I$ is the ideal $\operatorname{sat}_{x_{n}}(I)$ that is obtained from $I$ by setting $x_{n}=1$ in every monomial of $I$.

(iii) The double saturation of $I$ is the extension ideal $\operatorname{sat}_{x_{n-1}, x_{n}}(I)$ in $R$ of the saturation of $\operatorname{sat}_{x_{n}}(I) \cap R^{(1)} \subset R^{(1)}$. It is obtained from $I$ by setting $x_{n}=x_{n-1}=1$.

Throughout this paper we use the lexicographic order, $>_{l e x}$, for comparing monomials of a given degree. Let $x^{B}=x_{0}^{b_{0}} x_{1}^{b_{1}} \cdots x_{n}^{b_{n}}$ and $x^{C}=x_{0}^{c_{0}} x_{1}^{c_{1}} \cdots x_{n}^{c_{n}}$ be two monomials of $R$ of the same degree. Recall that $x^{B}>_{\text {lex }} x^{C}$, if the first nonzero entry of the vector $\left(b_{0}-c_{0}, b_{1}-c_{1}, \ldots, b_{n}-c_{n}\right)$ is positive.

If $A$ is a graded $K$-algebra we denote its Hilbert function by $h_{A}(j)=\operatorname{dim}_{K}[A]_{j}$, its Hilbert polynomial by $p_{A}$, and its Hilbert series by $H_{A}=\sum_{j \geq 0} h_{A}(j) \cdot t^{j}$. The Hilbert functions of graded $K$-algebras are completely classified. In particular, if $h$ is such a Hilbert function with $h(1) \leq n+1$, then there is a lexsegment ideal $L_{h} \subset R$ such that, for every integer $j, h_{R / L_{h}}(j)=h(j)$. Recall that a lexsegment ideal is a monomial ideal $I \subset R$ such that, for every integer $j,[I]_{j}$ is spanned by the first $\operatorname{dim}_{K}[I]_{j}$ monomials of $[R]_{j}$ in the lexicographic order. Lexsegment ideals are strongly stable.

At times we will abuse language and say that a homogeneous ideal $I$ of $R$ has Hilbert function $h$ or Hilbert polynomial $p$ if $R / I$ has this Hilbert function or polynomial.

Let $p \in \mathbb{Q}[z]$ be the Hilbert polynomial of a standard graded $K$-algebra of dimension $d+1>0$. Then there are unique integers $b_{0} \geq b_{1} \geq b_{2} \geq \cdots \geq b_{d}>0$ such that

$$
p(z)=\sum_{i=0}^{d}\left[\left(\begin{array}{c}
z+i \\
i+1
\end{array}\right)-\left(\begin{array}{c}
z+i-b_{i} \\
i+1
\end{array}\right)\right] .
$$

With respect to this representation, we recall the lexicographic ideal associated to a given Hilbert polynomial as introduced by Macaulay. Some of the properties of this ideal have been studied by Bayer in [1. The lexicographic ideal is called a universal lex ideal in [19] and [4]. In order to keep this paper more self-contained and for the convenience of the reader we provide short proofs for the results below. 
Theorem 2.3. Let $p \neq 0$ be a Hilbert polynomial of a quotient of $R$. Then there is a unique saturated lexsegment ideal $L_{p} \subset R$ such that the Hilbert polynomial of $R / L_{p}$ is $p$. It is called the lexicographic ideal to $p$. The ideal $L_{p}$ is generated by the set of monomials

$$
\begin{aligned}
& \left\{x_{0}, x_{1}, \ldots, x_{n-d-2}, x_{n-d-1}^{a_{d}+1}, x_{n-d-1}^{a_{d}} \cdot x_{n-d}^{a_{d-1}+1},\right. \\
& \quad x_{n-d-1}^{a_{d}} \cdot x_{n-d}^{a_{d-1}} \cdot x_{n-d+1}^{a_{d-2}+1}, \ldots, \\
& x_{n-d-1}^{a_{d}} \cdot x_{n-d}^{a_{d-1}} \cdot x_{n-d+1}^{a_{d-2}} \cdots \cdots x_{n-3}^{a_{2}} \cdot x_{n-2}^{a_{1}+1}, \\
& \left.x_{n-d-1}^{a_{d}} \cdot x_{n-d}^{a_{d-1}} \cdot x_{n-d+1}^{a_{d-2}} \cdots \cdots x_{n-2}^{a_{1}} \cdot x_{n-1}^{a_{0}}\right\},
\end{aligned}
$$

where $p$ is written as in equation (2.1) and $a_{d}:=b_{d}, a_{d-1}:=b_{d-1}-b_{d}, \ldots, a_{0}:=$ $b_{0}-b_{1}$ (thus, $\left.b_{i}=a_{d}+a_{d-1}+\ldots+a_{i}\right), 0 \leq i \leq d$.

Proof. Because of its importance and for the convenience of the reader we include a proof. Set $L\left(a_{0}, \ldots, a_{d}\right):=L_{p}$. It is clearly a lexsegment ideal and saturated. We use induction on $d \geq 0$ in order to compute the Hilbert polynomial of the quotient. If $d=0$, then we have $R / L\left(a_{0}\right)=K\left[x_{0}, \ldots, x_{n}\right] /\left(x_{0}, \ldots, x_{n-2}, x_{n-1}^{a_{0}}\right) \cong$ $K\left[x_{n-1}, x_{n}\right] /\left(x_{n-1}^{a_{0}}\right)$, and thus the Hilbert polynomial is $p_{R / L\left(a_{0}\right)}(z)=a_{0}=\left(\begin{array}{c}z \\ 1\end{array}\right)-$ $\left(\begin{array}{c}z-a_{0} \\ 1\end{array}\right)=p$, as claimed.

Let $d>0$. Then multiplication by $x_{n-d-1}^{a_{d}}$ provides the exact sequence

$$
\begin{gathered}
0 \longrightarrow\left(R / L\left(a_{0}, \ldots, a_{d-1}\right)\right)\left(-a_{d}\right) \stackrel{x_{n-d-1}^{a_{d}}}{\longrightarrow} R / L\left(a_{0}, \ldots, a_{d}\right) \\
\longrightarrow R /\left(x_{0}, \ldots, x_{n-d-2}, x_{n-d-1}^{a_{d}}\right) \longrightarrow 0 .
\end{gathered}
$$

Using the induction hypothesis we conclude that $p_{R / L\left(a_{0}, \ldots, a_{d}\right)}=p$.

The uniqueness statement follows from the fact that $L_{p}$ is a lexsegment ideal and saturated.

Note that the set of generators of the lexicographic ideal $L_{p}$ given in Theorem 2.3 is not minimal when $a_{0}=0$.

The ideal $L_{p}$ has alternative characterizations.

Proposition 2.4. (a) Let $L_{h} \subset R$ be a lexsegment ideal with Hilbert polynomial $p$, i.e., if $j \gg 0$, then $p(j)=h(j)$. Then the saturation of $L_{h}$ is the ideal $L_{p} \subset R$.

(b) Let $R / I$ be a graded quotient of $R$ with Hilbert polynomial $p$. Then, for all integers $j$ :

$$
h_{R / I}(j) \geq h_{L_{p}}(j) .
$$

Proof. (a) Since $L_{h}$ and $L_{p}$ are both lexsegment ideals and $h(j)=p(j)$ whenever $j \gg 0$, we get

$$
\left[L_{h}\right]_{j}=\left[L_{p}\right]_{j},
$$

whenever $j \gg 0$. As the ideal $L_{p}$ is saturated, it follows that $L_{p}$ is the saturation of $L_{h}$. (b) Denote by $h$ the Hilbert function of $R / I$. Then part (a) implies $L_{h} \subset L_{p}$, and the claim follows.

We conclude this section with formulae for certain invariants of stable ideals (in particular, strongly stable ideals), which will be useful later. Note that these invariants only depend on the max indices and the degrees of the minimal generators of the ideal. 
Remark 2.5. If $I \subset R$ is a saturated strongly stable ideal with minimal monomial generators $\left\{x^{A_{1}}, \ldots, x^{A_{r}}\right\}$, then let $l_{i}=\max \left(x^{A_{i}}\right)$ and $d_{i}=\operatorname{deg}\left(x^{A_{i}}\right)$, for all $1 \leq$ $i \leq r$. The Hilbert polynomial and (nonreduced) Hilbert series of $R / I$ are

$$
p_{R / I}(z)=\left(\begin{array}{c}
z+n \\
n
\end{array}\right)-\sum_{i=1}^{r}\left(\begin{array}{c}
z+n-d_{i}-l_{i} \\
n-l_{i}
\end{array}\right)
$$

and

$$
H_{R / I}(t)=\left(1-\sum_{i=1}^{r}(1-t)^{l_{i}} t^{d_{i}}\right)(1-t)^{-n-1} .
$$

The total Betti numbers of the ideal $I$ are

$$
\beta_{j}(I)=\sum_{i=1}^{r}\left(\begin{array}{l}
l_{i} \\
j
\end{array}\right) .
$$

Equations (2.3) and (2.4) follow from the Eliahou-Kervaire resolution for stable monomial ideals (see [7, p. 16); equation (2.2) is a direct consequence of (2.3).

\section{EXPANSIONS AND CONTRACTIONS OF MONOMIALS}

Throughout the remainder of this paper, $I \varsubsetneqq R=K\left[x_{0}, \ldots, x_{n}\right]$ always denotes a saturated strongly stable ideal and $G(I)$ the set of its minimal monomial generators. If $n \leq 1$, then these ideals are principal. Thus, it is harmless to assume $n \geq 2$. At times, we will abuse terminology by saying that $I$ has Hilbert polynomial $p$ if $p$ is actually the Hilbert polynomial of the quotient $R / I$.

We first define left-shifts and right-shifts for monomials, and then use left-shifts and right-shifts to define contractions and expansions of monomials. We adapt Reeves's definitions for left-shifts and right-shifts of monomials and for contractions of monomials (see 24] and Remarks 3.3(iii) and 3.8 below). Expansions will play a central role in the algorithm to compute all saturated strongly stable ideals to a given Hilbert polynomial.

Definition 3.1. Let $x^{A} \in R$ be a monomial of positive degree.

(i) The set of right-shifts of $x^{A}$ is

$$
\mathcal{R}\left(x^{A}\right):=\left\{x^{A} \frac{x_{i+1}}{x_{i}}: x_{i} \mid x^{A}, 0 \leq i<n-1\right\} .
$$

(ii) The set of left-shifts of $x^{A}$ is

$$
\mathcal{L}\left(x^{A}\right):=\left\{x^{A} \frac{x_{i-1}}{x_{i}}: x_{i} \mid x^{A}, 0<i \leq n-1\right\} .
$$

Example 3.2. Consider the monomial $x_{1}^{2} x_{3} \in K\left[x_{0}, \ldots, x_{5}\right]$. As its right-shifts we get

$$
\mathcal{R}\left(x_{1}^{2} x_{3}\right)=\left\{x_{1} x_{2} x_{3}, x_{1}^{2} x_{4}\right\} .
$$

For its left-shifts we obtain

$$
\mathcal{L}\left(x_{1}^{2} x_{3}\right)=\left\{x_{0} x_{1} x_{3}, x_{1}^{2} x_{2}\right\}
$$


Remark 3.3. (i) Observe that all monomials in $\mathcal{L}\left(x^{A}\right)$ and $\mathcal{R}\left(x^{A}\right)$ have the same degree as $x^{A}$. Furthermore, every monomial in $\mathcal{L}\left(x^{A}\right)$ is larger than $x^{A}$ in the lexicographic order, and every monomial in $\mathcal{R}\left(x^{A}\right)$ is less than $x^{A}$. In particular, $\mathcal{L}\left(x^{A}\right) \cap \mathcal{R}\left(x^{A}\right)=\varnothing$ and neither of the sets, $\mathcal{L}\left(x^{A}\right)$ nor $\mathcal{R}\left(x^{A}\right)$, contains the monomial $x^{A}$ itself.

(ii) The set of left-shifts of any monomial of the form $x_{0}^{k}$ is empty $\left(\mathcal{L}\left(x_{0}^{k}\right)=\varnothing\right)$. This fact will be important below.

(iii) The original definitions for left-shifts and right-shifts in [24] included redundant monomials. The above definitions provide the smallest sets which can be used to determine whether an ideal will continue to be strongly stable after adding or removing minimal monomial generators (see Lemma 3.9).

Next, we introduce expansion and contractions.

Definition 3.4. Let $x^{A}$ be a monomial of $R$.

(i) If $x^{A} \neq 1$ is a minimal generator of $I$ such that $G(I) \cap \mathcal{R}\left(x^{A}\right)=\varnothing$, then we call $x^{A}$ expandable in $I$ (or simply expandable if the ideal is understood). The expansion of $x^{A}$ in $I$ is defined to be the ideal $I^{\mathfrak{e x p}}$ generated by the set

$$
G\left(I^{\mathfrak{e} \mathfrak{p}}\right):=\left(G(I) \backslash\left\{x^{A}\right\}\right) \cup\left\{x^{A} \cdot x_{r}, x^{A} \cdot x_{r+1}, \ldots, x^{A} \cdot x_{n-1}\right\},
$$

where $r=\max \left(x^{A}\right)$.

If $I=R$ and $x^{A}=1$, then we set $I^{\mathfrak{e x p}}:=\left(x_{0}, \ldots, x_{n-1}\right)$.

(ii) If $x^{A} \neq 1$ is a monomial in $R$ such that $x^{A} \cdot x_{n-1} \in G(I)$ (so $\left.x^{A} \notin I\right)$ and $\mathcal{L}\left(x^{A}\right) \subset I$, then we call $x^{A}$ contractible in $I$ (or simply contractible if the ideal is understood). The contraction of $x^{A}$ in $I$ is defined to be the ideal $I^{\mathfrak{c o n}}$ generated by the set

$$
G\left(I^{\mathfrak{c o n}}\right):=\left(G(I) \cup\left\{x^{A}\right\}\right) \backslash\left\{x^{A} \cdot x_{r}, x^{A} \cdot x_{r+1}, \ldots, x^{A} \cdot x_{n-1}\right\},
$$

where $r=\max \left(x^{A}\right)$.

If $x_{n-1} \in G(I)$ and $x^{A}=1$, then we set $I^{\text {con }}:=(1)=R$.

We note that expandable monomials have been studied elsewhere as Borel generators.

Remark 3.5. The expandable monomials of a strongly stable ideal are exactly the Borel generators; compare our Definition [3.4(i) with Proposition 2.13 in [8. The introduction of [8] discusses several papers involving Borel generators.

Example 3.6. Consider the saturated strongly stable ideal $I:=\left(x_{0}^{3}, x_{0}^{2} x_{1}, x_{0}^{2} x_{2}\right) \subset$ $K\left[x_{0}, x_{1}, x_{2}, x_{3}\right]$. The monomial $x_{0}^{2} x_{2}$ is expandable in $I$ because the monomial in $\mathcal{R}\left(x_{0}^{2} x_{2}\right)=\left\{x_{0} x_{1} x_{2}\right\}$ is not a minimal generator of $I$. The expansion of $x_{0}^{2} x_{2}$ in $I$ is generated by

$$
G\left(I^{\mathfrak{e} \mathfrak{p}}\right)=G(I) \backslash\left\{x_{0}^{2} x_{2}\right\} \cup\left\{x_{0}^{2} x_{2}^{2}\right\}=\left\{x_{0}^{3}, x_{0}^{2} x_{1}, x_{0}^{2} x_{2}^{2}\right\} .
$$

Now the monomial $x_{0}^{2} x_{2}$ is contractible in $I^{\mathfrak{e} \mathfrak{w}}=\left(x_{0}^{3}, x_{0}^{2} x_{1}, x_{0}^{2} x_{2}^{2}\right)$ since it is not contained in $I^{\mathfrak{e x p}}$ and $\mathcal{L}\left(x_{0}^{2} x_{2}\right)=\left\{x_{0}^{2} x_{1}\right\}$ is in $I^{\mathfrak{e x p}}$. The contraction of $x_{0}^{2} x_{2}$ in $I^{\mathfrak{e} \mathfrak{p}}$ is the ideal $I$ we started with.

Similarly, the monomial $x_{0}^{2}$ is contractible in $I$ because it is not in the ideal, the monomial $x_{0}^{2} x_{2}$ is a minimal generator of $I$, and $\mathcal{L}\left(x_{0}^{2}\right)=\varnothing \subset I$. The contraction of $x_{0}^{2}$ in $I$ is generated by

$$
G\left(I^{\mathfrak{c o n}}\right)=G(I) \cup\left\{x_{0}^{2}\right\} \backslash\left\{x_{0}^{3}, x_{0}^{2} x_{1}, x_{0}^{2} x_{2}\right\}=\left\{x_{0}^{2}\right\} .
$$


Now the monomial $x_{0}^{2}$ is expandable in $I^{\mathfrak{c o n}}=\left(x_{0}^{2}\right)$ since it is the only minimal generator (so the set of right-shifts is automatically disjoint from the set of minimal generators of the ideal). The expansion of $x_{0}^{2}$ in $I^{\text {con }}$ is the ideal $I$ we started with.

As seen in this example, the contraction and expansion of a monomial in a saturated strongly stable ideal are inverse operations. This will be a useful fact.

Lemma 3.7. Let $x^{A} \in R$ be a monomial.

(a) If $x^{A}$ is expandable in $I$, then $x^{A}$ is contractible in the resulting expansion $I^{\mathfrak{e} \mathfrak{p}}$. The contraction of $x^{A}$ in $I^{\mathfrak{e x p}}$ is $I$.

(b) If $x^{A}$ is contractible in $I$, then $x^{A}$ is expandable in the resulting contraction $I^{\mathfrak{c o n}}$. The expansion of $x^{A}$ in $I^{\mathfrak{c o n}}$ is $I$.

Proof. These observations follow directly from Definition 3.4 .

Remark 3.8. Following 9], our Definition 3.4(ii) differs from Reeves's original definition in Appendix A.2 of 24 in two places as we insist on $x^{A} \cdot x_{n-1} \in G(I)$, but require only $\mathcal{L}\left(x^{A}\right) \subset I$ instead of $\mathcal{L}\left(x^{A}\right) \subset G(I)$. The first change is necessary for Lemma 3.7(ii); the second is essential to establish Lemma 3.11 (see also Example 3.12).

Contractions and expansions are defined so that they will produce saturated strongly stable ideals. The proof is straightforward, but is included nonetheless.

Lemma 3.9. If a monomial $x^{A}$ is contractible or expandable in $I$, then $I^{\text {con }}$ or $I^{\mathfrak{e x p}}$ is a saturated strongly stable ideal, respectively.

Proof. Note that if $I$ is saturated, then $I^{\mathfrak{c o n}}$ or $I^{\mathfrak{e} \mathfrak{p}}$ will by definition also be saturated.

Suppose that $x^{A}$ is contractible. By Remark 2.2(i), we need only show that $\left(x_{i} / x_{j}\right) \cdot x^{A} \in I^{\text {con }}$ for all $j$ such that $x_{j} \mid x^{A}$ and all $i<j$. Since $x^{A}$ is contractible, $\mathcal{L}\left(x^{A}\right) \subset I$. Thus, for all $j$ such that $x_{j} \mid x^{A}$, each monomial $\left(x_{j-1} / x_{j}\right) \cdot x^{A} \in I$ so the monomial is also in $I^{\mathfrak{c o n}}$. Because $I$ is strongly stable, if $\left(x_{j-1} / x_{j}\right) \cdot x^{A} \in I$, then $\left(x_{i} / x_{j}\right) \cdot x^{A} \in I$ for all $i<j$, so $\left(x_{i} / x_{j}\right) \cdot x^{A} \in I^{\text {con }}$ for all $i<j$.

Suppose $x^{A}$ is expandable. Now, we need to establish that we have a strongly stable ideal after removing the monomial $x^{A}$ from $G(I)$. Consider a monomial $x^{B}$ of the form $\left(x_{k} / x_{j}\right) \cdot x^{A}$ for some $j$ such that $x_{j} \mid x^{A}$ and $k>j$. Then the monomial $x^{B}$ is not in $I$, because $\left(x_{j+1} / x_{j}\right) \in \mathcal{R}\left(x^{A}\right), \mathcal{R}\left(x^{A}\right)$ is disjoint from $I$, and $I$ is strongly stable. Thus, the monomial $x^{A}$ can be removed from $G(I)$ without destroying strong stability.

In any saturated strongly stable ideal, there will always be expandable monomials. If the ideal is not doubly saturated, there will be contractible monomials. The particular expansions and contractions described in the following result form the basis for Section 5 .

Lemma 3.10. In any fixed degree, the minimal monomial generator of $I$, which is smallest according to the lexicographic order, will be expandable.

If the ideal I is not doubly saturated, then some minimal monomial generators will contain the variable $x_{n-1}$. In any fixed degree $d$, among the monomials $x^{A}$ of degree $d-1$ such that $x^{A} x_{n-1}$ is a minimal monomial generator of the ideal, the monomial, which is largest according to the lexicographic order, will be contractible.

Proof. These observations follow directly from Definition 3.4 and Remark 3.3(i). 
Our aim is to use expansions to produce saturated strongly stable ideals from simpler ideals-ideals with fewer minimal generators or minimal generators of smaller degree. We start with the following result, which appears as Lemma 23 in [24]. We follow Reeves's argument with some suitable modifications.

Lemma 3.11. There is a finite sequence of contractions taking the ideal $I$ to its double saturation $\operatorname{sat}_{x_{n-1}, x_{n}}(I)$.

Proof. Since $I$ is saturated, no minimal generators are divisible by $x_{n}$. Consider the set $M$ of monomials in $G(I)$ that are divisible by $x_{n-1}$. If $M=\varnothing$, then $I$ is doubly saturated. Otherwise, choose the monomial $x^{A} \cdot x_{n-1}$ of least degree in $M$, which is largest with respect to the lexicographic order $>_{\text {lex }}$. As noted in Lemma 3.10, $x^{A}$ is contractible in $I$.

Let $I^{\mathfrak{c o n}}$ be the contraction of $x^{A}$ in $I$. Note that contracting $x^{A}$ replaces $x^{A} \cdot x_{n-1}$ (and possibly other monomials) by $x^{A}$. Thus, $I^{\mathfrak{c o n}}$ has the same double saturation as $I$. After repeating the above step some finite number of times, we get an ideal whose minimal generators are not divisible by $x_{n-1}$. This is the double saturation of $I$.

Example 3.12. We illustrate the last proof with the ideal $I=\left(x_{0}, x_{1}^{2}, x_{1} x_{2}^{3}\right)$ in the ring $K\left[x_{0}, x_{1}, x_{2}, x_{3}\right]$.

- First we contract the monomial $x_{1} x_{2}^{2}$ in $I$. The resulting ideal $I_{1}$ is generated minimally by

$$
G\left(I_{1}\right)=G(I) \cup\left\{x_{1} x_{2}^{2}\right\} \backslash\left\{x_{1} x_{2}^{3}\right\}=\left\{x_{0}, x_{1}^{2}, x_{1} x_{2}^{2}\right\} .
$$

(Note that $\mathcal{L}\left(x_{1} x_{2}^{2}\right)=\left\{x_{0} x_{2}^{2}, x_{1}^{2} x_{2}\right\}$ is not a subset of the set of minimal generators of $I$. This shows that our modification of Reeves's definition of contraction in 24 is needed in the above argument.)

- Next, we contract $x_{1} x_{2}^{2}$ and get the ideal

$$
I_{2}=\left(x_{0}, x_{1}^{2}, x_{1} x_{2}\right) .
$$

- In the last step, contracting $x_{1}$ in $I_{2}$ gives the double saturation

$$
I_{3}=\left(x_{0}, x_{1}\right)=\operatorname{sat}_{x_{2}, x_{3}}(I) .
$$

We now make the contractions necessary to get to the double saturation more explicit.

Remark 3.13. Assume that the ideal $I$ is different from its double saturation. List the minimal generators of $I$ that are divisible by $x_{n-1}$,

$$
x^{A_{1}} x_{n-1}^{e_{1}}, x^{A_{2}} x_{n-1}^{e_{2}}, \ldots, x^{A_{s}} x_{n-1}^{e_{s}},
$$

where $x^{A_{i}}$ is not divisible by $x_{n-1}$, so that $\operatorname{deg} x^{A_{i}} x_{n-1}^{e_{i}} \leq \operatorname{deg} x^{A_{i+1}} x_{n-1}^{e_{i+1}}$, and in the case of equality $x^{A_{i}} x_{n-1}^{e_{i}}>_{\text {lex }} x^{A_{i+1}} x_{n-1}^{e_{i+1}}$. Then the contractions in the algorithm given in the proof of Lemma 3.11 use the following monomials

$$
x^{A_{1}} x_{n-1}^{e_{1}-1}, x^{A_{1}} x_{n-1}^{e_{1}-2}, \ldots, x^{A_{1}}, x^{A_{2}} x_{n-1}^{e_{2}-1}, \ldots, x^{A_{2}}, \ldots, x^{A_{s}}
$$

in the stated order. Thus, we need $e_{1}+e_{2}+\cdots+e_{s}$ contractions to compute the double saturation of $I$.

Since this process is reversible, we can recover an ideal from its double saturation: 
Corollary 3.14. There is a finite sequence of expansions taking the double saturation of an ideal $\operatorname{sat}_{x_{n-1}, x_{n}}(I)$ to the ideal I. In particular, the necessary number of expansions can be determined by adding up the exponents of $x_{n-1}$ in the minimal generators of $I$.

Proof. The sequence of contractions described in Remark 3.13, which take $I$ to its double saturation, can be reversed and considered as expansions by Lemma 3.7 .

We conclude this section by describing the change of the Hilbert function under contraction or expansion.

Lemma 3.15. (a) Let $I^{\mathfrak{e x p}}$ be the expansion of $x^{A}$ in $I$. Then

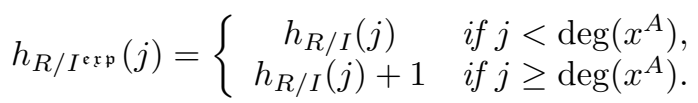

(b) Let $I^{\text {con }}$ be the contraction of $x^{B}$ in $I$. Then

$$
h_{R / I^{\mathfrak{c o n}}}(j)=\left\{\begin{array}{cl}
h_{R / I}(j) & \text { if } j<\operatorname{deg}\left(x^{B}\right), \\
h_{R / I}(j)-1 & \text { if } j \geq \operatorname{deg}\left(x^{B}\right) .
\end{array}\right.
$$

Proof. (a) We have $I^{\mathfrak{e} \mathfrak{x}} \subset I$. Furthermore, if $j \geq \operatorname{deg}\left(x^{A}\right)$, then $x^{A} \cdot x_{n}^{j-\operatorname{deg}\left(x^{A}\right)}$ is the only monomial in $[I]_{j} \backslash\left[I^{\mathfrak{e x p}}\right]_{j}$. The claim follows.

(b) Now, $I \subset I^{\text {con }}$, and $x^{B} \cdot x_{n}^{j-\operatorname{deg}\left(x^{B}\right)}$ is the only monomial in $\left[I^{\mathfrak{c o n}}\right]_{j} \backslash[I]_{j}$, provided $j \geq \operatorname{deg}\left(x^{B}\right)$.

We can now determine the number of expansions to recover an ideal from its double saturation in a more abstract manner.

Corollary 3.16. The number of expansions needed to take $J=\operatorname{sat}_{x_{n-1}, x_{n}}(I)$ to $I$ is

$$
p_{R / I}-p_{R / J}
$$

\section{Strongly stable ideals with a given Hilbert polynomial}

In this section, we describe how to produce all saturated strongly stable ideals with a given Hilbert polynomial. We develop a few more tools, which culminate in Theorem 4.4 and Algorithm 4.6. We start with the simplest case, ideals with constant Hilbert polynomial:

Lemma 4.1. Let $I \subset R$ be a saturated strongly stable ideal with constant Hilbert polynomial, say $p_{R / I}=c$. Then sat $_{x_{n-1}, x_{n}}(I)=(1)=R$. Moreover, any saturated strongly stable ideal $J \subset R$ with $p_{R / J}=c$ can be obtained from the ideal (1) using c suitable expansions.

Proof. If $x_{n-1}^{k} \in I$, then sat $x_{n-1}, x_{n}(I)=(1)=R$ by Remark 2.2(iii). Assume that no power of $x_{n-1}$ is in $I$. Let $j$ be any positive integer. Since $I$ is strongly stable, no monomial of the form $x_{n-1}^{j-i} \cdot x_{n}^{i} \in I$ for $0 \leq i \leq j$. Hence, there are at least $j+1$ monomials not contained in $[I]_{j}$ for every $j>0$, which contradicts $p_{R / I}(z)=c$. Thus, some power of $x_{n-1}$ is in $I$. The final claim is now a consequence of Corollaries 3.14 and 3.16 .

Recall some previously introduced notation: $R^{(j)}:=K\left[x_{0}, \ldots, x_{n-j}\right]$ is the polynomial ring where the last $j$ variables of $R$ have been removed. If $I \subset R$ is a saturated strongly stable ideal with Hilbert polynomial $p$, then the restriction of its 
double saturation $\operatorname{sat}_{x_{n-1}, x_{n}}(I)$ to $R^{(1)}:=K\left[x_{0}, \ldots, x_{n-1}\right]$ is a saturated strongly stable ideal in $R^{(1)}$ with a Hilbert polynomial that can be computed from $p$ :

Lemma 4.2. If $I$ is a saturated strongly stable ideal with Hilbert polynomial $p(z)$ and double saturation $J=\operatorname{sat}_{x_{n-1}, x_{n}}(I)$, then the Hilbert polynomial of $J^{(1)}:=$ $J \cdot R^{(1)} \subset R^{(1)}$ is $p_{R^{(1)} / J^{(1)}}(z)=\Delta p(z):=p(z)-p(z-1)$.

Proof. Setting $I^{(1)}=I \cdot R^{(1)}$, multiplication by $x_{n}$ induces the exact sequence

$$
0 \longrightarrow R / I(-1) \stackrel{x_{n}}{\longrightarrow} R / I \longrightarrow R^{(1)} / I^{(1)} \longrightarrow 0
$$

since $x_{n}$ is not a zero divisor of $R / I$. Now, $p_{R^{(1)} / I^{(1)}}(z)=\Delta p(z)$. Passing to $J^{(1)}$, the saturation of $I^{(1)}$, does not change the Hilbert polynomial, so $p_{R^{(1)} / J^{(1)}}(z)=$ $\Delta p(z)$.

This result can be extended. If $p(z)$ is a Hilbert polynomial of degree $d$, we set $\Delta^{0} p(z):=p(z)$, and recursively define $\Delta^{j} p(z):=\Delta^{j-1} p(z)-\Delta^{j-1} p(z-1)$ for $1 \leq j \leq d$. Thus, $\Delta=\Delta^{1}$. Now, if $I$ is a saturated strongly stable ideal, then, for $0 \leq j \leq d$, we denote by $I^{(j)} \subset R^{(j)}$, the saturated strongly stable ideal whose generating set is obtained by setting $x_{n-j}=\cdots=x_{n-1}=1$ in the monomial generators of $I$. Note that the ideal $I^{(j+1)} \cdot R^{(j)}$ is the double saturation of $I^{(j)}$. Repeating the argument in Lemma 4.2 shows that $\Delta^{j} p(z)$ is the Hilbert polynomial of the ideal $I^{(j)}$ :

Corollary 4.3. If $I$ is a saturated strongly stable ideal with Hilbert polynomial $p(z)$ of degree $d$, and $I^{(j)} \subset R^{(j)}$ is the ideal obtained by setting $x_{n-j}=\cdots=$ $x_{n-1}=1$ in the monomial generators of $I$, then the Hilbert polynomial of $I^{(j)}$ is $p_{R^{(j)} / I^{(j)}}(z)=\Delta^{j} p(z)$ for $0 \leq j \leq d$.

We are now ready to prove the main result of this section.

Theorem 4.4. Let $I \varsubsetneqq R$ be a saturated strongly stable ideal with Hilbert polynomial $p(z)$ of degree $\bar{d}$. Then there is a finite sequence of expansions (in the appropriate rings) that take the ideal $(1)=R^{(d)}$ to the ideal $I \subset R$.

In particular, the number of expansions needed in $R^{(j)}$ to take $I^{(j+1)} \cdot R^{(j)}$ to $I^{(j)}$ is $\Delta^{j} p(z)-p_{R^{(j)} / I^{(j+1)} R^{(j)}}(z)$, which, in the notation of Theorem 2.3 , is at most $a_{j}$, for $j=0, \ldots, d$. The total number of expansions needed to take $(1)=R^{(d)}$ to the ideal $I \subset R$ is at most $b_{0}$.

Proof. Let $p(z)$ be the Hilbert polynomial of $R / I$. We induct on the degree of $p(z)$. If $\operatorname{deg} p=0$, then we are done by Lemma 4.1. Assume $\operatorname{deg} p>0$. Since $\operatorname{deg} \Delta^{1} p=$ $\operatorname{deg} p-1$, we conclude by the induction hypothesis that there is a finite sequence of expansions that takes the ideal $(1) \subset R^{(d)}$ to $J^{(1)}=\operatorname{sat}_{x_{n-1}, x_{n}}(I) \subset R^{(1)}$, the double saturation of $I$ as an ideal in $R^{(1)}$. Considering the corresponding extension ideal in $R$, the ideal $I$ can be obtained from $J^{(1)} \cdot R$ by Corollary 3.14 using a finite number of expansions.

The claim that the number of expansions needed in the $\operatorname{ring} R^{(j)}$ to take the ideal $I^{(j+1)} \cdot R^{(j)}$ to the ideal $I^{(j)}$ is $\Delta^{j} p(z)-p_{R^{(j)} / I^{(j+1)} R^{(j)}}(z)$ follows from Corollaries 3.16 and 4.3 . Thus it remains to show that

$$
\Delta^{j} p(z)-p_{R^{(j)} / I^{(j+1)} R^{(j)}}(z) \leq a_{j}
$$

because the final assertion then follows by recalling that $b_{0}=a_{0}+\cdots+a_{d}$. 
In order to establish inequality (4.1) write the given Hilbert polynomial as in equation (2.1) as

$$
p(z)=\sum_{i=0}^{d}\left[\left(\begin{array}{c}
z+i \\
i+1
\end{array}\right)-\left(\begin{array}{c}
z+i-b_{i} \\
i+1
\end{array}\right)\right] .
$$

By Lemma 4.2, the Hilbert polynomial of $I^{(j)}$ is

$$
\begin{aligned}
p_{R^{(j)} / I^{(j)}}(z) & =\Delta^{j} p(z) \\
& =\sum_{i=j}^{d}\left[\left(\begin{array}{c}
z+i-j \\
i+1-j
\end{array}\right)-\left(\begin{array}{c}
z+i-b_{i}-j \\
i+1-j
\end{array}\right)\right] .
\end{aligned}
$$

Using Theorem 2.3, it follows that exactly $a_{j}$ expansions in $R^{(j)}$ are needed to take the lexicographic ideal $L_{\Delta^{j+1} p} R^{(j)}$ to the lexicographic ideal $L_{\Delta^{j} p}$ of $R^{(j)}$. Since $R^{(j+1)} / I^{(j+1)}$ and $R^{(j+1)} / L_{\Delta^{j+1} p}$ have the same Hilbert polynomial, namely $\Delta^{j+1} p$, inequality (4.1) is equivalent to

$$
p_{R^{(j)} / I^{(j+1)} R^{(j)}}(z) \geq p_{R^{(j)} / L_{\Delta^{j+1} p} R^{(j)}}(z) .
$$

(The difference of the two polynomials is a constant.) However, the latter estimate is a consequence of Proposition 2.4 (b) because $L_{\Delta^{j+1} p} \subset R^{(j+1)}$ is the saturation of $L_{\Delta^{j} p} R^{(j+1)}$ in $R^{(j+1)}$, so, for all integers $k$,

$$
h_{R^{(j+1)} / I^{(j+1)}}(k) \geq h_{R^{(j+1)} / L_{\Delta^{j+1} p}}(k) .
$$

Summing over $k$ on both sides of this inequality, we get the Hilbert functions of $R^{(j)} / I^{(j+1)} R^{(j)}$ and $R^{(j)} / L_{\Delta^{j+1} p} R^{(j)}$, respectively. Now, inequality (4.2) follows.

Note that the estimate on the number of needed expansions is sharp. This follows from Lemma 5.3 below.

The particular expansions leading to the lexicographic ideal $L_{p}$ can be made explicit.

Remark 4.5. In Theorem 4.4, the lexicographic ideal will be obtained if, at each step, the minimal monomial generator to be expanded is of the highest degree, and is smallest according to the lexicographic order in that degree. This follows by Proposition 2.4(b) and Lemma 3.10

Using Theorem 4.4 and its proof, we can now give the desired algorithm to compute all saturated strongly stable ideals with a prescribed Hilbert polynomial.

Algorithm 4.6 (Generating all saturated strongly stable ideals with a given Hilbert polynomial). Let $p(z)$ be a nonzero Hilbert polynomial of degree $d$ of a graded quotient of $R$.

(1) Compute the polynomials $\Delta^{1} p(z), \Delta^{2} p(z), \ldots, \Delta^{d} p(z)$. $\left(\right.$ Note that $\Delta^{d} p(z)=c$ for some $c \in \mathbb{N}$.) Set $\mathcal{S}^{(d)}=\cdots=\mathcal{S}^{(0)}=\varnothing$.

(2) Generate $\mathcal{S}^{(d)}$, the set of all saturated strongly stable ideals I in $R^{(d)}$ with Hilbert polynomial $p_{R^{(d)} / I}(z)=\Delta^{d} p(z)=c$, using $c$ successive expansions of monomial generators starting with the ideal $(1)=R^{(d)}$. Exhaust all choices for c successive expansions. 
(3) For $j=d-1, d-2, \ldots, 0$, repeat the following steps for each ideal $I \in \mathcal{S}^{(j+1)}$ :

Compute $p_{R^{(j)} / I}(z)$ (using equation (2.2)). Let $a=\Delta^{j} p(z)-p_{R^{(j)} / I}(z)$.

- If $a \geq 0$, then perform a successive expansions of monomial generators of I to obtain ideals with Hilbert polynomial $\Delta^{j} p(z)$. Exhaust all choices for a successive expansions. Add these ideals to $\mathcal{S}^{(j)}$.

- If a $<$, then continue with the next ideal I in $\mathcal{S}^{(j+1)}$.

(4) Return the set $\mathcal{S}^{(0)}$.

Proof (Correctness). By Theorem 4.4, every saturated strongly stable ideal with Hilbert polynomial $p(z)$ will be generated by this algorithm, as long as every possible sequence of expansions is carried out at each step. Also, every ideal generated by this process will be saturated and strongly stable and have the desired Hilbert polynomial.

The algorithm terminates for any given Hilbert polynomial, since the number of steps performed in (3) is bounded by the degree of the Hilbert polynomial and the number of generators in each ideal computed in each loop is finite.

Note that different algorithms to achieve the same goal have been proposed by Reeves in 24 and Cioffi, Lella, Marinari, and Roggero in 5]. We defer a comparison of these algorithms to Remark 7.2 .

When carrying out Algorithm 4.6, one can order the expansions so that each ideal is produced in a unique way.

Remark 4.7. One natural ordering of minimal generators is to always list the monomials first by degree in increasing order and then lexicographically in each degree. When expanding in some $\operatorname{ring} R^{(j)}$, always pick monomials, which precede all other monomials that have been expanded in this ring (those monomials divisible by the variable $x_{n-j-1}$ ). (Thus, the expanded monomials, leading to a certain ideal, will be strictly increasing according to this order and, hence, unique.) This is the reverse of the order for contractions discussed in Remark 3.13 .

We include an example to illustrate this algorithm.

Example 4.8. Suppose we wish to find all saturated strongly stable ideals with Hilbert polynomial $p(z)=\frac{3}{2} z^{2}+\frac{5}{2} z=\left(\begin{array}{c}z+2 \\ 3\end{array}\right)-\left(\begin{array}{c}z-1 \\ 3\end{array}\right)+\left(\begin{array}{c}z+1 \\ 2\end{array}\right)-\left(\begin{array}{c}z-3 \\ 2\end{array}\right)+\left(\begin{array}{c}z \\ 1\end{array}\right)-\left(\begin{array}{c}z-5 \\ 1\end{array}\right)$ in $R=K\left[x_{0}, x_{1}, x_{2}, x_{3}, x_{4}\right]$.

- First we compute $\Delta^{1} p(z)$ and $\Delta^{2} p(z)$ :

$$
\Delta^{1} p(z)=3 z+1, \quad \Delta^{2} p(z)=3 .
$$

- Next we generate all ideals in $R^{(2)}=K\left[x_{0}, x_{1}, x_{2}\right]$ with Hilbert polynomial $\Delta^{2} p(z)=3$ using 3 successive expansions and starting from $(1)=R^{(2)}$. We get two ideals:

$$
I=\left(x_{0}, x_{1}^{3}\right), \quad J=\left(x_{0}^{2}, x_{0} x_{1}, x_{1}^{2}\right) .
$$

- Now we generate all ideals in $R^{(1)}$ with Hilbert polynomial $\Delta^{1} p(z)=3 z+1$. We compute the Hilbert polynomials of $I$ and $J$ in $R^{(1)}$ :

$$
p_{R^{(1)} / I}(z)=3 z, \quad p_{R^{(1)} / J}(z)=3 z+1 .
$$

We perform one expansion in $I$ to obtain the following ideals:

$$
I_{1}=\left(x_{0}, x_{1}^{4}, x_{1}^{3} x_{2}\right), \quad I_{2}=\left(x_{0}^{2}, x_{0} x_{1}, x_{0} x_{2}, x_{1}^{3}\right) .
$$


We perform no expansions in $J$ (as it already has the desired Hilbert polynomial).

- Finally, we generate all ideals in $R$ with Hilbert polynomial $p(z)=\frac{3}{2} z^{2}+\frac{5}{2} z$. We compute the Hilbert polynomials of $I_{1}, I_{2}$, and $J$ :

$$
\begin{gathered}
p_{R / I_{1}}(z)=\frac{3}{2} z^{2}+\frac{5}{2} z-1, \quad p_{R / I_{2}}(z)=\frac{3}{2} z^{2}+\frac{5}{2} z+1, \\
p_{R / J}(z)=\frac{3}{2} z^{2}+\frac{5}{2} z+1 .
\end{gathered}
$$

We ignore the ideals $I_{2}$ and $J$ because their Hilbert polynomials in $R$ are too large. We perform one expansion in $I_{1}$ to obtain the following ideals:

$$
\left(x_{0}, x_{1}^{4}, x_{1}^{3} x_{2}^{2}, x_{1}^{3} x_{2} x_{3}\right), \quad\left(x_{0}^{2}, x_{0} x_{1}, x_{0} x_{2}, x_{0} x_{3}, x_{1}^{4}, x_{1}^{3} x_{2}\right) .
$$

Thus, there are two saturated strongly stable ideals in $R$ with Hilbert polynomial $p(z)=\frac{3}{2} z^{2}+\frac{5}{2} z$. Note that $\left(x_{0}, x_{1}^{4}, x_{1}^{3} x_{2}^{2}, x_{1}^{3} x_{2} x_{3}\right)$ is the lexicographic ideal.

As illustrated in the example above, it is possible to generate extraneous ideals in the intermediate steps of Algorithm 4.6. By Lemma 3.15] and Remark 4.5, the Hilbert function of the lexicographic ideal will be not greater in each degree than the Hilbert function of any other ideal produced by the algorithm. When determining the Hilbert function of an ideal in a ring with another variable, the new Hilbert function in degree $d$ is the sum of the previous Hilbert function in degrees up to $d$. Thus, expanding in too small of a degree (compared to the lexicographic ideal) may increase the Hilbert polynomial by too much. However, a few computer experiments suggest that this extra effort to avoid the computation of extraneous ideals is not worth it.

It is possible to directly produce only the desired ideals by following a different procedure; see the end of Remark 7.2 .

\section{Almost lexsegment ideals with a given Hilbert polynomial}

In this section, we develop an algorithm for producing a unique ideal for each Hilbert series associated to a given Hilbert polynomial. This algorithm is helpful when looking for ideals with a fixed Hilbert polynomial, which have maximal Betti numbers.

We begin by introducing the class of strongly stable ideals in which we are now interested. If a strongly stable ideal is saturated, then no minimal monomial generators contain the last variable $x_{n}$. Thus, the ideal can be considered in the polynomial ring $R^{(1)}$, where the variable $x_{n}$ has been removed. This class of ideals is characterized by the fact that they are lexsegment ideals when viewed in the smaller ring $R^{(1)}$.

Definition 5.1. A saturated strongly stable ideal $I \subset R$ is called almost lexsegment if $I \cdot R^{(1)}$ is a lexsegment ideal.

Example 5.2. Consider the saturated strongly stable ideals $I_{1}=\left(a^{2}, a b, a c, b^{2}\right)$, $I_{2}=\left(a^{2}, a b, a c, b^{3}, b^{2} c\right)$, and $I_{3}=\left(a^{2}, a b, a c^{2}, b^{3}, b c^{2}\right)$ in $R=K[a, b, c, d] . I_{1}, I_{2}$ and $I_{3}$ are almost lexsegment ideals. $I_{1}$ is generated by the first four monomials of $R^{(1)}=K[a, b, c]$ in degree two. $I_{2}$ contains the first three monomials of $R^{(1)}$ in degree two, the first seven monomials of $R^{(1)}$ in degree three, etc; $I_{3}$ contains the first two monomials of $R^{(1)}$ in degree two, the first eight monomials of $R^{(1)}$ in degree three, etc. 
We will now focus on characterizing how to generate almost lexsegment ideals. The process will be similar to the previous algorithm, except for two simplifications: all lexsegment ideals have the same double saturation and are produced by certain expansions.

Recall the lexicographic ideal $L_{p}$ and the nonnegative integers $a_{i}$ introduced earlier in Theorem 2.3. which are associated to each Hilbert polynomial.

Lemma 5.3. Every almost lexsegment ideal with Hilbert polynomial $p(z)$ has the same double saturation, namely $L_{\widetilde{p}}$, where

$$
\widetilde{p}(z)=p(z)-a_{0} .
$$

Proof. Using the definition of $\widetilde{p}$, we see that the ideal $L_{\widetilde{p}}$ is doubly saturated by Theorem 2.3 (because no minimal generator will be divisible by $x_{n-1}$ ). Thus, the ideal $L_{\widetilde{p}} \cdot R^{(1)} \subset R^{(1)}$ is the unique saturated lexsegment ideal of $R^{(1)}$ with Hilbert polynomial $\Delta p(z)$ by Lemma 4.2 .

The double saturation of an almost lexsegment ideal $I \subset R$ with Hilbert polynomial $p(z)$ will also be a saturated lexsegment ideal in $R^{(1)}$ with Hilbert polynomial $\Delta p(z)$. Thus, the double saturation must be $L_{\widetilde{p}}$.

Note that the uniqueness statement of the double saturation in Lemma 5.3 is equivalent to Proposition 2.3 in [4]. The explicit description of the double saturation is new.

We give a name to the special expansions and contractions that were noted earlier in Lemma 3.10

Definition 5.4. Let $I \subset R$ be an almost lexsegment ideal.

(i) In any fixed degree, an expansion of the minimal monomial generator of $I$, which is last according to the lexicographic order, is called a lex expansion.

(ii) In any fixed degree, a contraction of the monomial $x^{A}$ such that $x^{A} x_{n-1}$ is a minimal monomial generator, which is first according to the lexicographic order, is called a lex contraction.

Note that lex expansions and lex contractions are inverse operations. (For any lex expansion, there is a lex contraction which will undo it, and vice versa.)

Lex expansions and lex contractions are the only tools needed to produce almost lexsegment ideals:

Lemma 5.5. If I is an almost lexsegment ideal, then applying a lex expansion or a lex contraction to I will produce another almost lexsegment ideal.

In fact, the only expansions of almost lexsegment ideals which produce almost lexsegment ideals are the lex expansions, and, similarly, the only contractions of almost lexsegment ideals which produce almost lexsegment ideals are the lex contractions.

Proof. Assume $I \subset R$ is an almost lexsegment ideal.

Expanding a monomial $x^{A}$ of degree $d$ only changes the ideal $I \cdot R^{(1)}$ in degree $d$ (by removing the monomial $x^{A}$ from $I$ ). If $x^{A}$ is the smallest minimal monomial generator of $I$ in degree $d$ according to the lexicographic order, then the expansion of $x^{A}$ will be an almost lexsegment ideal. Expanding a monomial of degree $d$ which comes before $x^{A}$ in the lexicographic order will produce an ideal which is not almost lexsegment. 
Similarly, contracting a monomial $x^{A}$ of degree $d$ only changes the ideal $I \cdot R^{(1)}$ in degree $d$ (by adding the monomial $x^{A}$ ). If $x^{A}$ is the largest minimal monomial generator of $I$ in degree $d$ according to the lexicographic order, then the contraction of $x^{A}$ will be an almost lexsegment ideal. Contracting a monomial of degree $d$ which comes after $x^{A}$ in the lexicographic order will produce an ideal which is not almost lexsegment.

We illustrate the last lemma with an example.

Example 5.6. Consider again the almost lexsegment ideals $I_{1}=\left(a^{2}, a b, a c, b^{2}\right)$, $I_{2}=\left(a^{2}, a b, a c, b^{3}, b^{2} c\right)$, and $I_{3}=\left(a^{2}, a b, a c^{2}, b^{3}, b c^{2}\right)$ in $R=K[a, b, c, d]$ from Example 5.2 .

Observe that the smallest monomial generator in $I_{1}$ of degree two, according to the lexicographic order, is $b^{2}$. This monomial is expandable, and expanding it produces the almost lexsegment ideal $I_{2}$. The monomial $a c$ is also expandable in $I_{1}$, but expanding it produces an ideal, $J=\left(a^{2}, a b, a c^{2}, b^{2}\right)$, which is not almost lexsegment (since $a c$ is not in $J, b^{2}$ is in $J$, and $a c>_{\text {lex }} b^{2}$ ).

Observe that there are two contractible monomials in $I_{3}: a c$ and $b^{2}$. As $a c$ is greater than $b^{2}$ in the lexicographic order, contracting $a c$ produces the almost lexsegment ideal $I_{2}$, while contracting $b^{2}$ produces the ideal $J$, which is not almost lexsegment.

We summarize the above results:

Corollary 5.7. Each almost lexsegment ideal with Hilbert polynomial $p(z)$ can be obtained from its double saturation $L_{\widetilde{p}}$ through a sequence of $a_{0}$ lex expansions (exclusively) through almost lexsegment ideals.

Proof. If an almost lexsegment ideal is not doubly saturated, then we can perform a lex contraction to produce another almost lexsegment ideal. Repeating a finite number of times will yield the double saturation. Since lex expansions and lex contractions are inverse operations, we can go in the other direction.

The number of needed expansions is $a_{0}$ by Lemma 5.3 and Corollary 3.16.

Combining Corollaries 3.16 and 5.7 yields the following procedure.

Algorithm 5.8 (Generating all almost lexsegment ideals with a given Hilbert polynomial). Let $p(z)$ be a nonzero Hilbert polynomial of some graded quotient of $R$.

(1) Compute $a_{0}$ from $p(z)$ and the double saturation of the lexicographic ideal, $L_{\widetilde{p}}$ (as in Theorem 2.3), where $\widetilde{p}(z)=p(z)-a_{0}$.

(2) Perform $a_{0}$ successive lex expansions of monomial generators of $L_{\widetilde{p}}$. Exhaust all choices for $a_{0}$ successive lex expansions.

The following example illustrates this process.

Example 5.9. Suppose we wish to find all almost lexsegment ideals with Hilbert polynomial $p(z)=2 z^{2}+z+1$ in $R=K\left[x_{0}, x_{1}, x_{2}, x_{3}, x_{4}\right]$.

- First we compute the double saturation of the lexicographic ideal for $p$. The lexicographic ideal is $\left(x_{0}, x_{1}^{5}, x_{1}^{4} x_{2}^{2}, x_{1}^{4} x_{2} x_{3}^{2}\right)$ so

$$
L_{\widetilde{p}}=\left(x_{0}, x_{1}^{5}, x_{1}^{4} x_{2}\right) .
$$

Note that $a_{0}=2$. 
- Next we make two lex expansions in all possible ways to produce the following four almost lexsegment ideals with the desired Hilbert polynomial:

$$
\begin{gathered}
\left(x_{0}, x_{1}^{5}, x_{1}^{4} x_{2}^{2}, x_{1}^{4} x_{2} x_{3}^{2}\right), \quad\left(x_{0}, x_{1}^{6}, x_{1}^{5} x_{2}, x_{1}^{5} x_{3}, x_{1}^{4} x_{2}^{2}, x_{1}^{4} x_{2} x_{3}\right), \\
\left(x_{0}^{2}, x_{0} x_{1}, x_{0} x_{2}, x_{0} x_{3}, x_{1}^{5}, x_{1}^{4} x_{2}^{2}, x_{1}^{4} x_{2} x_{3}\right), \quad\left(x_{0}^{2}, x_{0} x_{1}, x_{0} x_{2}, x_{0} x_{3}^{2}, x_{1}^{5}, x_{1}^{4} x_{2}\right) .
\end{gathered}
$$

As noted in the introduction, Caviglia and Murai [4] recently showed that there is a saturated ideal which achieves maximal total Betti numbers among all ideals with a given Hilbert polynomial. By a result of Bigatti, Hulett and Pardue, it is enough to consider almost lexsegment ideals when looking for ideals with maximal Betti numbers. Using Algorithm 5.8, one can determine all such ideals.

Remark 5.10. If one only wants to produce the almost lexsegment ideals with maximal Betti numbers, suitable modifications significantly reduce the number of ideals that are produced in Algorithm 5.8. In fact, at the beginning of the algorithm it is enough to repeatedly expand all monomial of least degree in the ideal as many times as possible. The justification for this modification requires very different techniques and will appear in a forthcoming paper.

Caviglia and Murai note in their paper 4 that their proof "is very long and complicated" and their construction "is not easy to understand". Examples 5.11 and 5.12 show that there can be more than one ideal with maximal Betti numbers. A simpler construction or proof could perhaps be found by choosing a different set of ideals. This motivates the questions: How many ideals attain maximal Betti numbers and how can they be distinguished?

One idea is to consider the Hilbert function of the ideals in question. Because the ideals are almost lexsegment, their Hilbert functions will be distinct. One might hope that among all ideals with maximal Betti numbers, there is one which has a Hilbert function which is either larger in all degrees than the other Hilbert functions, or which is smaller in all degrees. Unfortunately, the following two examples show that this is not the case.

Notice however, that, by a result of Valla in [27, among the almost lexsegment ideals with a constant Hilbert polynomial and maximal Betti numbers, there is one ideal with a maximal Hilbert function. Such an ideal does not exist if the Hilbert polynomial has positive degree.

Example 5.11. In the polynomial ring $K\left[x_{0}, x_{1}, x_{2}, x_{3}, x_{4}\right]$, there are 509 saturated strongly stable ideals with Hilbert polynomial $p(z)=z^{2}+5 z+3$. Of these, 129 are almost lexsegment ideals, and four ideals attain maximal Betti numbers. All four ideals are obtained by making two lex expansions in the ideal

$$
\begin{array}{r}
\left(x_{0}^{3}, x_{0}^{2} x_{1}, x_{0}^{2} x_{2}, x_{0}^{2} x_{3}, x_{0} x_{1}^{2}, x_{0} x_{1} x_{2}, x_{0} x_{1} x_{3}, x_{0} x_{2}^{2}, x_{0} x_{2} x_{3}, x_{0} x_{3}^{2},\right. \\
\left.x_{1}^{4}, x_{1}^{3} x_{2}, x_{1}^{3} x_{3}, x_{1}^{2} x_{2}^{3}\right) .
\end{array}
$$

To maximize the Hilbert function, we want to expand in the smallest degree possible, but we have two choices: either we expand $x_{0} x_{3}^{2}$ and $x_{1}^{2} x_{2}^{3}$ (to maximize the Hilbert function in degree three) to obtain the ideal

$$
\begin{array}{r}
\left(x_{0}^{3}, x_{0}^{2} x_{1}, x_{0}^{2} x_{2}, x_{0}^{2} x_{3}, x_{0} x_{1}^{2}, x_{0} x_{1} x_{2}, x_{0} x_{1} x_{3}, x_{0} x_{2}^{2}, x_{0} x_{2} x_{3}, x_{0} x_{3}^{3},\right. \\
\left.x_{1}^{4}, x_{1}^{3} x_{2}, x_{1}^{3} x_{3}, x_{1}^{2} x_{2}^{4}, x_{1}^{2} x_{2}^{3} x_{3}\right),
\end{array}
$$


or we expand $x_{1}^{3} x_{2}$ and $x_{1}^{3} x_{3}$ (to maximize the Hilbert function in degree four) to obtain

$$
\begin{array}{r}
\left(x_{0}^{3}, x_{0}^{2} x_{1}, x_{0}^{2} x_{2}, x_{0}^{2} x_{3}, x_{0} x_{1}^{2}, x_{0} x_{1} x_{2}, x_{0} x_{1} x_{3}, x_{0} x_{2}^{2}, x_{0} x_{2} x_{3}, x_{0} x_{3}^{2},\right. \\
\left.x_{1}^{4}, x_{1}^{3} x_{2}^{2}, x_{1}^{3} x_{2} x_{3}, x_{1}^{3} x_{3}^{2}, x_{1}^{2} x_{2}^{3}\right) .
\end{array}
$$

The Hilbert functions of these two ideals are incomparable.

Minimal Hilbert functions among the ideals with maximal Betti numbers do not exist even in the case of a constant Hilbert polynomial.

Example 5.12. In the polynomial ring $K\left[x_{0}, x_{1}, x_{2}, x_{3}\right]$, there are 6,481 saturated strongly stable ideals with Hilbert polynomial $p(z)=31$. Of these, 2,649 are almost lexsegment ideals, and five ideals attain maximal Betti numbers. All five ideals are obtained by making eleven lex expansions in the ideal

$$
\left(x_{0}, x_{1}, x_{2}\right)^{4} \text {. }
$$

To minimize the Hilbert function, we want to expand in the largest degree possible, but we have two choices: either we expand the last nine monomials in degree four and expand the last monomial in the largest degree twice more (to minimize the Hilbert function in degree four) to obtain the ideal

$$
\begin{array}{r}
\left(x_{0}^{4}, x_{0}^{3} x_{1}, x_{0}^{3} x_{2}, x_{0}^{2} x_{1}^{2}, x_{0}^{2} x_{1} x_{2}, x_{0}^{2} x_{2}^{2}, x_{0} x_{1}^{4}, x_{0} x_{1}^{3} x_{2}, x_{0} x_{1}^{2} x_{2}^{2}, x_{0} x_{1} x_{2}^{3},\right. \\
\left.x_{0} x_{2}^{4}, x_{1}^{5}, x_{1}^{4} x_{2}, x_{1}^{3} x_{2}^{2}, x_{1}^{2} x_{2}^{3}, x_{1} x_{2}^{4}, x_{2}^{7}\right),
\end{array}
$$

or we expand the last six monomials in degree four and the last five monomials in degree six (to minimize the Hilbert function in degree five) to obtain

$$
\begin{array}{r}
\left(x_{0}^{4}, x_{0}^{3} x_{1}, x_{0}^{3} x_{2}, x_{0}^{2} x_{1}^{2}, x_{0}^{2} x_{1} x_{2}, x_{0}^{2} x_{2}^{2}, x_{0} x_{1}^{3}, x_{0} x_{1}^{2} x_{2}, x_{0} x_{1} x_{2}^{2}, x_{0} x_{2}^{3},\right. \\
\left.x_{1}^{6}, x_{1}^{5} x_{2}, x_{1}^{4} x_{2}^{2}, x_{1}^{3} x_{2}^{3}, x_{1}^{2} x_{2}^{4}, x_{1} x_{2}^{5}, x_{2}^{6}\right) .
\end{array}
$$

The Hilbert functions of these two ideals are incomparable.

\section{Strongly stable ideals with a given Hilbert Series}

We now present an algorithm for producing all saturated strongly stable ideals with a fixed Hilbert series. This process is similar to the procedure for producing the lexsegment ideal for a prescribed Hilbert series. In that procedure, one simply adds monomial generators, in the appropriate degree, according to the lexicographic order until the desired Hilbert series is obtained. We adapt this strategy by adding any monomial generator, in the appropriate degree, which yields another saturated strongly stable ideal. However, we make several observations to simplify this process and to make it easier to implement.

Monomial generators will be added to an ideal in order of increasing degree: generators in lowest degree will be added first, starting with a power of the variable $x_{0}$ (because if a principal ideal is strongly stable, it must be generated by a power of $x_{0}$ ) and ending with the generators of highest degree.

For each saturated strongly stable ideal $I$, we maintain a list, $L_{I}$, of the monomials which can be added to the generators of $I$, so that the resulting ideal is strongly stable. We also record the "remaining portion" of the numerator of the Hilbert series, $f_{I}(t)=\sum_{i=0}^{r} C_{i} t^{i}$, using equation (2.3). We always add monomials of degree $s d\left(f_{I}\right)=\min \left\{i: C_{i} \neq 0\right\}$, the smallest degree for which there is a nonzero coefficient in $f_{I}(t)$. Recall our notation $l_{A}=\max \left(x^{A}\right)$ for the max index of the monomial $x^{A}$ and $d_{A}=\operatorname{deg}\left(x^{A}\right)$ for the degree of the monomial $x^{A}$. 
To ensure that each saturated strongly stable ideal is created in a unique way, monomial generators are added lexicographically.

Algorithm 6.1 (Computation of all saturated strongly stable ideals with a given Hilbert series). Let $g(t)$ be the numerator of the nonreduced Hilbert series of a graded quotient of $R$.

(1) Set $\mathcal{S}=\mathcal{M}=\varnothing$. Compute $f_{(0)}(t)=1-g(t)$ and $s d\left(f_{(0)}\right)$. Add the ideal $I=\left(x_{0}^{s d\left(f_{(0)}\right)}\right)$ to $\mathcal{M}$. Update $f_{I}(t)$ to $f_{(0)}(t)-t^{s d\left(f_{(0)}\right)}$, compute $s d\left(f_{I}\right)$, and set $L_{I}$ to $\left\{x_{0}^{s d\left(f_{(0)}\right)-1} x_{1}^{s d\left(f_{I}\right)-s d\left(f_{(0)}\right)+1}\right\}$.

(2) Repeat until $\mathcal{M}$ is empty. Choose an ideal $I \in \mathcal{M}$. Do one of the following:

- If $f_{I}(t)=0$, remove the ideal I from $\mathcal{M}$ and add it to $\mathcal{S}$.

- If $L_{I}=\varnothing$, remove I from $\mathcal{M}$ and continue with the next ideal in $\mathcal{M}$.

- If $f_{I}(t) \neq 0$ and $L_{I} \neq \varnothing$, remove $I$ from $\mathcal{M}$ and replace it with the $\left|L_{I}\right|$ ideals obtained by adding a single monomial $x^{B}$ from $L_{I}$ to the generators of $I$. For each ideal $J_{B}$ added to $\mathcal{M}$, which is generated by $G(I) \cup\left\{x^{B}\right\}$ : update $f_{J_{B}}(t)$ to $f_{I}(t)-(1-t)^{l_{B}} t^{d_{B}}$, compute $s d\left(f_{J_{B}}\right)$, and set $L_{J_{B}}$ to $\left\{x^{A} x_{l_{A}}^{\left.\text {sd( } f_{J_{B}}\right)-d_{A}}: x^{A} \in L_{I}, x^{A} \neq x^{B}\right\}$. Do the following:

- If $l_{B}<n-1$ and $\mathcal{L}\left(\frac{x_{l_{B}+1}}{x_{l_{B}}} x^{B}\right) \subset I$, include $x^{B} x_{l_{B}}^{\left.\text {sd(f } f_{J}\right)-d_{B}-1} x_{l_{B}+1}$ in $L_{J_{B}}$.

- If $x_{l_{B}-1} \mid x^{B}$ and $\mathcal{L}\left(\frac{x_{l_{B}}}{x_{l_{B}-1}} x^{B}\right) \subset I$, include $x^{B} x_{l_{B}-1}^{-1} x_{l_{B}}^{s d\left(f_{J}\right)-d_{B}+1}$ in $L_{J_{B}}$.

(3) Return the set of ideals $\mathcal{S}$.

Proof (Correctness). Certainly, any ideal produced by the above process will be strongly stable (because we check that the ideal generated by $G(I) \cup\left\{x^{B}\right\}$ is strongly stable before adding the monomial $x^{B}$ to $L_{I}$ ) and saturated (because no monomials added to the set of generators will be divisible by the variable $x_{n}$ ), and it will have the desired Hilbert series (because the ideal is added to $\mathcal{S}$ when the Hilbert series is correct).

We need to show that every saturated strongly stable ideal is produced: specifically, for each ideal $I$ produced in the algorithm, $L_{I}$ contains every monomial $x^{B}$ which can be added (in the lexicographic order) to the ideal $I$ to produce a saturated strongly stable ideal, say $J$, generated by $G(I) \cup\left\{x^{B}\right\}$. Suppose that the ideal $J$ is strongly stable; then

$$
\left\{\frac{x_{i}}{x_{j}} x^{B}: x_{j} \mid x^{B}, i<j\right\} \subset I, \text { so, in particular, } x^{A}=\frac{x_{l_{B}-1}}{x_{l_{B}}} x^{B} \in I .
$$

Note that the monomial $x^{A}$ is the smallest monomial in the lexicographic order (in degree $d_{B}$ ), which must be contained in the ideal $I$ if $J$ is strongly stable. Turning this around, at most two new monomials, say $x^{E}$ and $x^{F}$, can be added to the generators of $I$ after the monomial $x^{A}$ :

$$
\left.x^{E}=\frac{x_{l_{A}+1}}{x_{l_{A}}} x^{A}\left(\text { if } l_{A}<n-1\right) \text { and } x^{F}=\frac{x_{l_{A}}}{x_{l_{A}-1}} x^{A} \text { (if } x_{l_{A}-1} \mid x^{A}\right) .
$$

These monomials, $x^{E}$ and $x^{F}$, are precisely those which are included in $L_{I}$. The monomials $x^{E}$ and $x^{F}$ are added to $L_{I}$, provided that $\mathcal{L}\left(x^{E}\right) \subset I$ or $\mathcal{L}\left(x^{F}\right) \subset I$ so that the ideals generated by $G(I) \cup\left\{x^{E}\right\}$ and $G(I) \cup\left\{x^{F}\right\}$ are saturated and strongly stable. Thus, every monomial $x^{B}$, which can be added to the generators 
of an ideal $I$ to produce a saturated strongly stable ideal, appears in $L_{I}$, so the algorithm will generate all of the desired ideals.

The algorithm terminates for any given Hilbert series because each list $L_{I}$ is finite and, by [10, there is an upper bound for the largest degree of a minimal generator of a saturated ideal that depends only on its Hilbert polynomial, which in turn is determined by the Hilbert series.

When carrying out Algorithm 6.1, one can produce the desired ideals in a unique way.

Remark 6.2. We are adding generators in increasing degrees, so we should only add a monomial $x^{B}$ to an ideal $I$ if $x^{B}$ is smaller in the lexicographic order than all of the monomials in $G(I)$ of the same degree. If the monomial generators of $I$ are listed in decreasing order in each degree, then we need only compare with the last generator of degree $d_{B}$. Thus, monomials will be added to the ideal by degree and lexicographically in each degree.

We include an example to illustrate this algorithm.

Example 6.3. Suppose we wish to find all saturated strongly stable ideals in $R=K\left[x_{0}, x_{1}, x_{2}, x_{3}, x_{4}\right]$ with Hilbert series $H_{R / I}(t)=\frac{1-6 t^{2}+8 t^{3}-3 t^{4}}{(1-t)^{5}}$. Thus, the numerator of the Hilbert series is $1-6 t^{2}+8 t^{3}-3 t^{4}$.

- We begin with the zero ideal. We compute $f_{(0)}(t)=6 t^{2}-8 t^{3}+3 t^{4}$ and $s d\left(f_{(0)}\right)=2$ (because $6 t^{2}$ is the smallest nonzero term in $\left.f_{(0)}\right)$. We add $I_{1}=\left(x_{0}^{2}\right)$ to $\mathcal{M}$, update $f_{I_{1}}(t)$ to $f_{(0)}(t)-t^{2}=5 t^{2}-8 t^{3}+3 t^{4}$, record $s d\left(f_{I_{1}}\right)=2$, and set $L_{I_{1}}$ to $\left\{x_{0} x_{1}\right\}$.

- We replace $I_{1}$ in $\mathcal{M}$ with a new ideal $I_{2}=\left(x_{0}^{2}, x_{0} x_{1}\right)$. We update $f_{I_{2}}$ to $f_{I_{1}}(t)-(1-t) t^{2}=4 t^{2}-7 t^{3}+3 t^{4}$, record $s d\left(f_{I_{2}}\right)=2$, and set $L_{I_{2}}$ to $\left\{x_{0} x_{2}, x_{1}^{2}\right\}$.

- We replace $I_{2}$ in $\mathcal{M}$ with the two ideals $I_{3}=\left(x_{0}^{2}, x_{0} x_{1}, x_{0} x_{2}\right)$ and $I_{4}=$ $\left(x_{0}^{2}, x_{0} x_{1}, x_{1}^{2}\right)$.

$-f_{I_{3}}=f_{I_{2}}(t)-(1-t)^{2} t^{2}=3 t^{2}-5 t^{3}+2 t^{4}, s d\left(f_{I_{3}}\right)=2$, and $L_{I_{3}}=$ $\left\{x_{0} x_{3}, x_{1}^{2}\right\}$

$-f_{I_{4}}=f_{I_{2}}(t)-(1-t) t^{2}=3 t^{2}-6 t^{3}+3 t^{4}, s d\left(f_{I_{4}}\right)=2$, and $L_{I_{4}}=\varnothing$ (because $x_{0} x_{2}>_{\text {lex }} x_{1}^{2}$ and $x_{0} x_{2} \notin I_{4}$ so $x_{1} x_{2}$ cannot be added to $I_{4}$ ).

- We replace $I_{3}$ in $\mathcal{M}$ with the two ideals $I_{5}=\left(x_{0}^{2}, x_{0} x_{1}, x_{0} x_{2}, x_{0} x_{3}\right)$ and $I_{6}=\left(x_{0}^{2}, x_{0} x_{1}, x_{0} x_{2}, x_{1}^{2}\right)$. We ignore $I_{4}$ (because $\left.L_{I_{4}}=\varnothing\right)$.

$-f_{I_{5}}=f_{I_{3}}(t)-(1-t)^{3} t^{2}=2 t^{2}-2 t^{3}-t^{4}+t^{5}, s d\left(f_{I_{5}}\right)=2$, and $L_{I_{5}}=\left\{x_{1}^{2}\right\}$;

$-f_{I_{6}}=f_{I_{3}}(t)-(1-t) t^{2}=2 t^{2}-4 t^{3}+2 t^{4}, s d\left(f_{I_{6}}\right)=2$, and $L_{I_{6}}=\left\{x_{1} x_{2}\right\}$.

- We replace $I_{5}$ in $\mathcal{M}$ with the ideal $I_{7}=\left(x_{0}^{2}, x_{0} x_{1}, x_{0} x_{2}, x_{0} x_{3}, x_{1}^{2}\right)$, and we replace $I_{6}$ with the ideal $I_{8}=\left(x_{0}^{2}, x_{0} x_{1}, x_{0} x_{2}, x_{1}^{2}, x_{1} x_{2}\right)$.

$-f_{I_{7}}=f_{I_{5}}(t)-(1-t) t^{2}=t^{2}-t^{3}-t^{4}+t^{5}, s d\left(f_{I_{7}}\right)=2$, and $L_{I_{7}}=\left\{x_{1} x_{2}\right\}$;

$-f_{I_{8}}=f_{I_{6}}(t)-(1-t)^{2} t^{2}=t^{2}-2 t^{3}+t^{4}, s d\left(f_{I_{8}}\right)=2$, and $L_{I_{8}}=\left\{x_{2}^{2}\right\}$.

- We replace $I_{7}$ in $\mathcal{M}$ with the ideal $I_{9}=\left(x_{0}^{2}, x_{0} x_{1}, x_{0} x_{2}, x_{0} x_{3}, x_{1}^{2}, x_{1} x_{2}\right)$, and we replace $I_{8}$ with the ideal $I_{10}=\left(x_{0}^{2}, x_{0} x_{1}, x_{0} x_{2}, x_{1}^{2}, x_{1} x_{2}, x_{2}^{2}\right)$.

$-f_{I_{9}}=f_{I_{7}}(t)-(1-t)^{2} t^{2}=t^{3}-2 t^{4}+t^{5}, s d\left(f_{I_{9}}\right)=3$, and $L_{I_{9}}=\left\{x_{1} x_{3}^{2}, x_{2}^{3}\right\}$ (because we need to add monomials of degree 3 );

$-f_{I_{10}}=f_{I_{8}}(t)-(1-t)^{2} t^{2}=0$ (we do not need $s d\left(f_{I_{10}}\right)$ or $\left.L_{I_{10}}\right)$. 
- We add $I_{10}$ to $\mathcal{S}$, and we replace $I_{9}$ in $\mathcal{M}$ with the two ideals $I_{11}=$ $\left(x_{0}^{2}, x_{0} x_{1}, x_{0} x_{2}, x_{0} x_{3}, x_{1}^{2}, x_{1} x_{2}, x_{1} x_{3}^{2}\right)$ and $I_{12}=\left(x_{0}^{2}, x_{0} x_{1}, x_{0} x_{2}, x_{0} x_{3}, x_{1}^{2}\right.$, $\left.x_{1} x_{2}, x_{2}^{3}\right)$.

$-f_{I_{11}}=f_{I_{9}}(t)-(1-t)^{3} t^{3}=t^{4}-2 t^{5}+t^{6}, s d\left(f_{I_{11}}\right)=4$, and $L_{I_{11}}=\left\{x_{2}^{4}\right\}$;

- $f_{I_{12}}=f_{I_{9}}(t)-(1-t)^{2} t^{3}=0$ (we do not need $s d\left(f_{I_{12}}\right)$ or $\left.L_{I_{12}}\right)$.

- We add $I_{12}$ to $\mathcal{S}$, and we replace $I_{11}$ in $\mathcal{M}$ with the ideal $I_{13}=$ $\left(x_{0}^{2}, x_{0} x_{1}, x_{0} x_{2}, x_{0} x_{3}, x_{1}^{2}, x_{1} x_{2}, x_{1} x_{3}^{2}, x_{2}^{4}\right)$.

$-f_{I_{13}}=f_{I_{11}}(t)-(1-t)^{2} t^{4}=0$.

- We add $I_{13}$ to $\mathcal{S}$.

Thus, there are three saturated strongly stable ideals with the given Hilbert series:

$$
\begin{gathered}
\left(x_{0}^{2}, x_{0} x_{1}, x_{0} x_{2}, x_{1}^{2}, x_{1} x_{2}, x_{2}^{2}\right), \\
\left(x_{0}^{2}, x_{0} x_{1}, x_{0} x_{2}, x_{0} x_{3}, x_{1}^{2}, x_{1} x_{2}, x_{2}^{3}\right), \\
\left(x_{0}^{2}, x_{0} x_{1}, x_{0} x_{2}, x_{0} x_{3}, x_{1}^{2}, x_{1} x_{2}, x_{1} x_{3}^{2}, x_{2}^{4}\right) .
\end{gathered}
$$

This algorithm can in fact be used to produce all strongly stable ideals with a given Hilbert series.

Remark 6.4. As noted in Remark 2.2(ii), the monomial generators of a saturated strongly ideal will not be divisible by the last variable. Thus, to produce all strongly stable ideals in the ring $K\left[x_{0}, \ldots, x_{n}\right]$ with a given Hilbert series, $H(t)$, one could simply produce all saturated strongly ideals in the ring $K\left[x_{0}, \ldots, x_{n}, x_{n+1}\right]$ with the Hilbert series

$$
H(t) \cdot \frac{1}{1-t}
$$

using Algorithm 6.1 and consider them as ideals in the original ring.

\section{Applications and Related questions}

We conclude by discussing some questions that, we believe, deserve further investigation along with some initial results.

It is well known that saturated strongly stable ideals figure prominently in the combinatorial structure of the Hilbert scheme (see, e.g., [25]). This motivates the following problem.

Question 7.1. What is the number of saturated strongly stable ideals in $R$ with a given Hilbert polynomial $p$ ?

Is there an explicit formula or a generating function for this number that depends only on $p$ and the number of variables in $R$ ?

In an appendix to her thesis [24, Reeves presents an algorithm for generating saturated strongly stable ideals with a given Hilbert polynomial. Also, another algorithm was proposed independently in a recent paper [5] by Cioffi, Lella, Marinari, and Roggero. We thank the authors for kindly pointing this out to us after we submitted the first version of this paper. We briefly discuss the differences between these algorithms.

Remark 7.2. Algorithm 4.6 differs from the algorithm presented by Reeves in 24]: Her algorithm first computes all Hilbert series associated to the desired Hilbert polynomial by pairs of contractions and expansions and then generates all saturated strongly stable ideals for each Hilbert series. A single Hilbert series or ideal may be generated a number of times in each of these steps. On the other hand, our 
algorithm directly creates all ideals, each in a unique way, building them in larger and larger rings. We also give direct methods for producing all Hilbert series to a particular Hilbert polynomial in Algorithm 5.8 and all saturated strongly stable ideals with a particular Hilbert series in Algorithm 6.1 that appear more efficient.

Furthermore, Reeves uses special matrices to encode the set of monomial generators of a strongly stable ideal. On these matrices, a certain kind of elementary row operation is performed to compute other saturated strongly stable ideals with the same Hilbert series. One problem to be solved then is that the correspondence between such matrices encoding strongly stable ideals and the set of strongly stable ideals itself (in a fixed polynomial ring) is not a bijection. The elementary row operations used may produce matrices, which do not encode any saturated strongly stable ideal. Hence, one needs a special procedure within the algorithm to check whether or not a given matrix represents a saturated strongly stable ideal. To avoid this trial and error technique, we did not use these matrices.

The algorithm suggested in [5] is more similar to Algorithm 4.6 in that it is recursive in the number of variables (and the degree of the Hilbert polynomial). However, instead of increasing the degrees of the minimal generators to achieve the correct Hilbert polynomial, a number of new generators are added to make the Hilbert function as large as possible in a fixed degree. Certain generators are then removed in all possible combinations to produce the desired saturated strongly stable ideals.

Observe that our approach has the advantage of allowing us to estimate the number of steps to produce an ideal with a given Hilbert polynomial (see Theorem 4.4).

Moreover, all saturated strongly stable ideals with a given Hilbert polynomial can be generated by combining Algorithms [5.8 and 6.1 Simply find all of the almost lexsegment ideals with the specified Hilbert polynomial (these will correspond uniquely to the Hilbert series of saturated ideals associated to the Hilbert polynomial) and then create all of the saturated strongly stable ideals for each Hilbert series. In particular, this approach will not produce any unnecessary ideals.

In Table 1 we present some experimental results for the number of strongly stable ideals with a given Hilbert polynomial in a given polynomial ring. Recall that the Hilbert polynomial is actually the Hilbert polynomial of the quotient by the ideal.

Table 1 illustrates that, by fixing the Hilbert polynomial, the number of strongly stable ideals in a polynomial ring with $n+1$ variables having this Hilbert polynomial increases with $n$ initially until it becomes stable and independent of $n$. This is indicated by the rightmost column in the table.

Our next result explains this observation.

Proposition 7.3. If $p(z)$ is a Hilbert polynomial, written as in equation (2.1), then the number of saturated strongly stable ideals with Hilbert polynomial $p(z)$ in $R=K\left[x_{0}, \ldots, x_{n}\right]$ is the same whenever $n \geq b_{0}+d-1$.

Proof. The first expansion, the expansion of 1 in $R^{(d)}$, gives $\left(x_{0}, \ldots, x_{n-d-1}\right)$, an ideal with $n-d$ variables. By Theorem 4.4 the number of the remaining expansions will be at most $b_{0}-1$ (and depends on how the expansions are chosen). It follows that the max index of any expanded monomial is at least $n-d-b_{0}+1$. Hence, if $b_{0}-1 \leq n-d$, then the number of saturated strongly stable ideals generated is not constrained by the number of variables. 
TABLE 1. The number of saturated strongly stable ideals with a given Hilbert polynomial, $p(z)$, in $K\left[x_{0}, \ldots, x_{n}\right]$ for several values of $n$

\begin{tabular}{|c|c|c|c|c|c|c|}
\hline$p(z)$ & $a_{0}, a_{1}, a_{2}$ & $n=3$ & $n=6$ & $n=9$ & $n=12$ & $n \gg 0$ \\
\hline 4 & $4,0,0$ & 3 & 3 & 3 & 3 & 3 \\
\hline 8 & $8,0,0$ & 12 & 19 & 20 & 20 & 20 \\
\hline 12 & $12,0,0$ & 44 & 104 & 117 & 119 & 119 \\
\hline 16 & $16,0,0$ & 143 & 504 & 617 & 640 & 644 \\
\hline 20 & $20,0,0$ & 425 & 2262 & 3034 & 3223 & 3271 \\
\hline 24 & $24,0,0$ & 1193 & 9578 & 14140 & 15425 & 15818 \\
\hline $4 z+2$ & $4,4,0$ & 14 & 28 & 28 & 28 & 28 \\
\hline $4 z+6$ & $8,4,0$ & 94 & 394 & 433 & 434 & 434 \\
\hline $4 z+10$ & $12,4,0$ & 469 & 3702 & 4536 & 4627 & 4632 \\
\hline $4 z+14$ & $16,4,0$ & 1939 & 27486 & 37792 & 39462 & 39677 \\
\hline $8 z-16$ & $4,8,0$ & 10 & 18 & 18 & 18 & 18 \\
\hline $8 z-12$ & $8,8,0$ & 66 & 213 & 232 & 233 & 233 \\
\hline $8 z-8$ & $12,8,0$ & 347 & 1911 & 2268 & 2310 & 2313 \\
\hline $8 z-4$ & $16,8,0$ & 1576 & 14490 & 18812 & 19510 & 19607 \\
\hline $2 z^{2}+6$ & $4,0,4$ & 3 & 18 & 19 & 19 & 19 \\
\hline $2 z^{2}+10$ & $8,0,4$ & 12 & 224 & 268 & 271 & 271 \\
\hline $2 z^{2}+14$ & $12,0,4$ & 44 & 2073 & 2835 & 2930 & 2938 \\
\hline $2 z^{2}+18$ & $16,0,4$ & 143 & 15883 & 24927 & 26468 & 26687 \\
\hline $2 z^{2}+4 z-12$ & $4,4,4$ & 14 & 45 & 46 & 46 & 46 \\
\hline $2 z^{2}+4 z-8$ & $8,4,4$ & 94 & 776 & 868 & 872 & 872 \\
\hline $2 z^{2}+4 z-4$ & $12,4,4$ & 469 & 9165 & 11417 & 11636 & 11649 \\
\hline $2 z^{2}+8 z-46$ & $4,8,4$ & 10 & 37 & 38 & 38 & 38 \\
\hline $2 z^{2}+8 z-42$ & $8,8,4$ & 66 & 588 & 667 & 671 & 671 \\
\hline $2 z^{2}+8 z-38$ & $12,8,4$ & 347 & 6535 & 8281 & 8464 & 8476 \\
\hline $4 z^{2}-16 z+40$ & $4,0,8$ & 3 & 18 & 19 & 19 & 19 \\
\hline $4 z^{2}-16 z+44$ & $8,0,8$ & 12 & 224 & 268 & 271 & 271 \\
\hline $4 z^{2}-16 z+48$ & $12,0,8$ & 44 & 2073 & 2835 & 2930 & 2938 \\
\hline $4 z^{2}-12 z+6$ & $4,4,8$ & 14 & 45 & 46 & 46 & 46 \\
\hline $4 z^{2}-12 z+10$ & $8,4,8$ & 94 & 761 & 853 & 857 & 857 \\
\hline $4 z^{2}-12 z+14$ & $12,4,8$ & 469 & 8662 & 10851 & 11069 & 11082 \\
\hline $4 z^{2}-8 z-44$ & $4,8,8$ & 10 & 37 & 38 & 38 & 38 \\
\hline $4 z^{2}-8 z-40$ & $8,8,8$ & 66 & 588 & 667 & 671 & 671 \\
\hline $4 z^{2}-8 z-36$ & $12,8,8$ & 347 & 6523 & 8269 & 8452 & 8464 \\
\hline
\end{tabular}

The bound on the number of variables given in the last result is optimal in some cases.

Example 7.4. (i) Fix integers $d \geq 0$ and $b_{0} \geq 1$. Consider the saturated strongly stable ideals $I$ of $R=K\left[x_{0}, \ldots, x_{n}\right]$ with Hilbert polynomial

$$
p(z)=\left(\begin{array}{c}
z+d \\
d
\end{array}\right)+b_{0}-1 .
$$

Then, using the notation of Theorem 2.3, $a_{0}=b_{0}-1, a_{1}=\cdots=a_{d-1}=0$, and $a_{d}=1$. Following Algorithm 4.6 the first expansion will produce the ideal 
$I^{(d)}=\left(x_{0}, \ldots, x_{n-d-1}\right) \subset R^{(d)}$. The remaining $b_{0}-1$ expansions all occur in $R$. If $n=b_{0}+d-1$, then expanding all of the $n-d=b_{0}-1$ variables will produce a saturated strongly stable ideal with the desired Hilbert polynomial that is generated by quadrics. However, if $n \leq b_{0}+d-2$, then any $b_{0}-1$ expansions of $I^{(d)}$ will produce an ideal having a minimal generator whose degree is at least 3 . Hence the bound on $n$ in Proposition 7.3 is optimal for this Hilbert polynomial.

(ii) Not every Hilbert polynomial will achieve this bound. Consider $p(z)=$ $3 z\left(=\left(\begin{array}{c}z+1 \\ 2\end{array}\right)-\left(\begin{array}{c}z-2 \\ 2\end{array}\right)+\left(\begin{array}{c}z \\ 1\end{array}\right)-\left(\begin{array}{c}z-3 \\ 1\end{array}\right)\right)$. If $n \geq 2$, there is exactly one saturated strongly stable ideal for this Hilbert polynomial even though $b_{0}+d-1=3$. (The Hilbert polynomial of the ideal generated by $\left(x_{0}, \ldots, x_{n-3}, x_{n-2}^{3}\right)$ is $p(z)=3 z$, while the Hilbert polynomial of the ideal $\left(x_{0}, \ldots, x_{n-4}, x_{n-3}^{2}, x_{n-3} x_{n-2}, x_{n-2}^{2}\right)$ is $3 z+1 \neq$ $p(z)$.)

It is known that the lexicographic ideal has the largest Castelnuovo-Mumford regularity among all saturated ideals with a fixed Hilbert polynomial (see [10, [1], and [25]). Theorem 4.4 provides a quick new argument. It also allows us to discuss the extremal ideals. We denote by gin $I$ the generic initial ideal of the ideal $I$ with respect to the reverse lexicographic order.

Theorem 7.5. Let $I \neq R$ be a saturated homogenous ideal of $R$. Write the Hilbert polynomial, $p$, of $R / I$ as in equation (2.1). Then the Castelnuovo-Mumford regularity of I satisfies

$$
\operatorname{reg} I \leq b_{0} .
$$

Furthermore, if $I$ is strongly stable, then equality is true if and only if $I=L_{p}$.

Moreover, if $I$ is any saturated homogenous ideal and char $K=0$, then $\operatorname{reg} I=b_{0}$ if and only if gin $I=L_{P}$ and $I$ is of the form

$$
I=\left(l_{0}, \ldots, l_{n-d-2}, f_{d} l_{n-d-1}, f_{d} f_{d-1} l_{n-d}, \ldots, f_{d} \ldots f_{t+1} l_{n-t-2}, f_{d} \ldots f_{t}\right)
$$

where $0 \leq t \leq d$, every $f_{i} \neq 0$ is a homogenous polynomial of degree $a_{i} \geq 0, a_{n}, a_{t} \geq$ 1 , every $l_{i}$ is a linear form, and $I$ has (as indicated) $n+1-t$ minimal generators. (Note that when $n=t$ the ideal $I$ is simply defined as $I=\left(f_{d}\right)$.)

Proof. First, we show the claims when $I$ is a strongly stable ideal. The EliahouKervaire resolution shows that the regularity of $I$ is the maximal degree of a minimal generator of $I$. By Theorem 4.4 we know that $I$ can be obtained from the ideal $(1)=R^{(d)}$ by at most $b_{0}$ expansions. Since each expansion replaces a monomial by monomials whose degree is one more, it follows immediately that the degrees of the minimal generators of $I$ are at most $b_{0}$.

In order to characterize equality we use induction on $b_{0} \geq 1$. If $b_{0}=1$, then $I$ is generated by linear forms, and the claim follows. Let $b_{0}>1$, and assume that $I$ has a minimal generator of degree $b_{0}$. Then, by the above argument, $I$ must have been obtained from the ideal $(1)=R^{(d)}$ by exactly $b_{0}$ expansions. Denote by $J^{\prime}$ the ideal obtained by the first $b_{0}-1$ expansions, and put $J=J^{\prime} R$. Then $J$ must have a minimal generator of degree $b_{0}-1$. Write the Hilbert polynomial of $R / J$ as

$$
p^{\prime}(z)=\sum_{i=0}^{d}\left[\left(\begin{array}{c}
z+i \\
i+1
\end{array}\right)-\left(\begin{array}{c}
z+i-b_{i}^{\prime} \\
i+1
\end{array}\right)\right] .
$$

Then $b_{0}^{\prime}=b_{0}-1$. Hence, the induction hypothesis provides that $J=L_{p^{\prime}}$. It follows that among the minimal generators of $J^{\prime}$ having degree $b_{0}-1$ only the smallest one 
in the lexicographic order is expandable. Expanding it, we get $I=L_{p}$ (see Remark 4.5).

Second, let $I$ be an arbitrary saturated homogenous ideal with the given Hilbert polynomial. Passing from $I$ to the almost lexsegment ideal $I^{*}$ with the same Hilbert function as $I$ can only increase the regularity by a result of Bigatti, Hulett, and Pardue (see [3], 18, [23]). Since almost lexsegment ideals are strongly stable we get $\operatorname{reg} I \leq \operatorname{reg} I^{*} \leq b_{0}$.

Finally, assume that the base field $K$ has characteristic zero. Then gin $I$ is strongly stable and has the same regularity as $I$ by [2]. Hence, by the first part of the proof, $\operatorname{reg} I=b_{0}$ if and only if $\operatorname{gin} I=L_{p}$. The claimed description of $I$ in this case now follows by Theorem 4.4 and Lemma 3.4 in [21].

Combined with the main result of Murai and Hibi in 20, we obtain the following consequence. We would like to thank Jeff Mermin for pointing this out.

Recall that a homogeneous ideal $I$ of $R=K\left[x_{0}, \ldots, x_{n}\right]$ is a Gotzmann ideal (see [16) if it has as many minimal generators as the lexsegment ideal $L_{h} \subset R$ corresponding to the Hilbert function of $I$. Notice that a strongly stable ideal $I$ of $R$ is saturated if it has at most $n$ minimal generators.

Corollary 7.6. Let $I \subset R$ be a saturated homogeneous ideal, where char $K=$ 0 . Write the Hilbert polynomial of $R / I$ as in equation (2.1). Then the following conditions are equivalent:

(a) $\operatorname{reg} I=b_{0}$;

(b) gin $I$ is a lexicographic ideal;

(c) I is a Gotzmann ideal with at most $n$ minimal generators;

(d) I is an ideal of the form as specified in equation (7.1).

Proof. Conditions (a), (b), and (d) are equivalent by Theorem 7.5 . The equivalence to condition (c) follows by Theorem 1.1 in 20 because (d) shows that $I$ is a canonical critical ideal up to a coordinate transformation.

We conclude with a crude estimate on the number of strongly stable ideals with a given Hilbert polynomial.

Corollary 7.7. Let $p$ be the Hilbert polynomial of a graded quotient of $R$. Using the notation of Theorem 2.3 , put $c=\min \left\{n, b_{0}+d-1\right\}$. Then the number of saturated strongly stable ideals in $R$ with Hilbert polynomial $p$ is at most

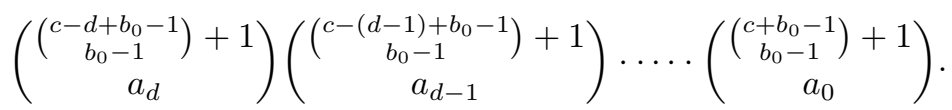

Proof. Assume first that $n \leq b_{0}+d-1$, that is, $c=n$.

Using the notation of Theorem 4.4 it takes at most $a_{j}$ expansions to take $I^{(j+1)}$. $R^{(j)}$ to $I^{(j)}$. By Theorem 7.5 , the degree of each expanded monomial is at most $b_{0}-1$. Moreover, we expand only monomials in $K\left[x_{0}, \ldots, x_{n-j-1}\right]$. There are $N_{j}=\left(\begin{array}{c}n-j+b_{0}-1 \\ b_{0}-1\end{array}\right)$ such monomials whose degree is at most $b_{0}-1$. For expanding at most $a_{j}$ of them, there are at most

$$
\left(\begin{array}{c}
N_{j} \\
0
\end{array}\right)+\left(\begin{array}{c}
N_{j} \\
1
\end{array}\right)+\cdots+\left(\begin{array}{c}
N_{j} \\
a_{j}
\end{array}\right)=\left(\begin{array}{c}
N_{j}+1 \\
a_{j}
\end{array}\right)
$$

possibilities. Since we take $I^{(j+1)}$ to $I^{(j)}$ for $j=d, d-1, \ldots, 0$, the claim follows in this case. 
Second, if $n \geq b_{0}+d-1$, then the number of strongly stable ideals is the same as for $n=b_{0}+d-1$ by Proposition 7.3. This concludes the argument.

For a constant Hilbert polynomial $p=C$ and $n \geq C-1$, the above bound is $\left(\begin{array}{c}\left(\begin{array}{c}2 C-2 \\ C-1\end{array}\right)+1 \\ C\end{array}\right)$. This is optimal if $C=1$. However, for $C=4$, the bound becomes $\left(\begin{array}{c}21 \\ 4\end{array}\right)=$ 5985 , whereas there are only three strongly stable ideals with Hilbert polynomial $p=4$.

\section{REFERENCES}

[1] David Allen Bayer, The Division Algorithm and the Hilbert Scheme, ProQuest LLC, Ann Arbor, MI, 1982. Thesis (Ph.D.)-Harvard University. MR2632095

[2] David Bayer and Michael Stillman, A criterion for detecting m-regularity, Invent. Math. 87 (1987), no. 1, 1-11, DOI 10.1007/BF01389151. MR862710 (87k:13019)

[3] Anna Maria Bigatti, Upper bounds for the Betti numbers of a given Hilbert function, Comm. Algebra 21 (1993), no. 7, 2317-2334, DOI 10.1080/00927879308824679. MR.1218500 (94c:13014)

[4] G. Caviglia, S. Murai, Sharp upper bounds of the Betti numbers for a given Hilbert polynomial, Algebra Number Theory, to appear

[5] Francesca Cioffi, Paolo Lella, Maria Grazia Marinari, and Margherita Roggero, Segments and Hilbert schemes of points, Discrete Math. 311 (2011), no. 20, 2238-2252, DOI 10.1016/j.disc.2011.07.011. MR.2825669 (2012j:14004)

[6] David Eisenbud, Commutative algebra: With a view toward algebraic geometry, Graduate Texts in Mathematics, vol. 150, Springer-Verlag, New York, 1995. MR.1322960 (97a:13001)

[7] Shalom Eliahou and Michel Kervaire, Minimal resolutions of some monomial ideals, J. Algebra 129 (1990), no. 1, 1-25, DOI 10.1016/0021-8693(90)90237-I. MR1037391(91b:13019)

[8] Christopher A. Francisco, Jeffrey Mermin, and Jay Schweig, Borel generators, J. Algebra 332 (2011), 522-542, DOI 10.1016/j.jalgebra.2010.09.042. MR2774702 (2012h:13033)

[9] K. Gehrs, On stable monomial ideals, Diploma Thesis, University of Paderborn, 2003.

[10] Gerd Gotzmann, Eine Bedingung für die Flachheit und das Hilbertpolynom eines graduierten Ringes, Math. Z. 158 (1978), no. 1, 61-70 (German). MR.0480478 (58 \#641)

[11] D. Grayson, M. Stillman, Macaulay2, a software system for research in algebraic geometry, Available at http://www.math.uiuc.edu/Macaulay2/.

[12] Mark L. Green, Generic initial ideals, Six lectures on commutative algebra (Bellaterra, 1996), Progr. Math., vol. 166, Birkhäuser, Basel, 1998, pp. 119-186. MR1648665 (99m:13040)

[13] Mark Green, Restrictions of linear series to hyperplanes, and some results of Macaulay and Gotzmann, Algebraic curves and projective geometry (Trento, 1988), Lecture Notes in Math., vol. 1389, Springer, Berlin, 1989, pp. 76-86, DOI 10.1007/BFb0085925. MR.1023391 (90k:13021)

[14] Robin Hartshorne, Connectedness of the Hilbert scheme, Inst. Hautes Études Sci. Publ. Math. 29 (1966), 5-48. MR 0213368 (35 \#4232)

[15] Robin Hartshorne, Algebraic geometry, Springer-Verlag, New York, 1977. Graduate Texts in Mathematics, No. 52. MR0463157(57 \#3116)

[16] Jürgen Herzog and Takayuki Hibi, Componentwise linear ideals, Nagoya Math. J. 153 (1999), 141-153. MR:1684555 (2000i:13019)

[17] Jürgen Herzog and Takayuki Hibi, Monomial ideals. Grad. Texts in Math. 260, Springer, 2011.

[18] Heather A. Hulett, Maximum Betti numbers of homogeneous ideals with a given Hilbert function, Comm. Algebra 21 (1993), no. 7, 2335-2350, DOI 10.1080/00927879308824680. MR.1218501 (94c:13015)

[19] Satoshi Murai and Takayuki Hibi, The depth of an ideal with a given Hilbert function, Proc. Amer. Math. Soc. 136 (2008), no. 5, 1533-1538, DOI 10.1090/S0002-9939-08-09067-9. MR2373580 (2009b:13028)

[20] Satoshi Murai and Takayuki Hibi, Gotzmann ideals of the polynomial ring, Math. Z. 260 (2008), no. 3, 629-646, DOI 10.1007/s00209-007-0293-2. MR2434473 (2010f:13025) 
[21] Uwe Nagel, Comparing Castelnuovo-Mumford regularity and extended degree: the borderline cases, Trans. Amer. Math. Soc. 357 (2005), no. 9, 3585-3603, DOI 10.1090/S0002-9947-0403595-0. MR2146640 (2006h:13027)

[22] R. Notari and M. L. Spreafico, A stratification of Hilbert schemes by initial ideals and applications, Manuscripta Math. 101 (2000), no. 4, 429-448, DOI 10.1007/s002290050225. MR:1759253(2001b:14008)

[23] Keith Pardue, Deformation classes of graded modules and maximal Betti numbers, Illinois J. Math. 40 (1996), no. 4, 564-585. MR1415019 (97g:13029)

[24] Alyson April Reeves, Combinatorial structure on the Hilbert scheme, ProQuest LLC, Ann Arbor, MI, 1992. Thesis (Ph.D.)-Cornell University. MR2688239

[25] Alyson A. Reeves, The radius of the Hilbert scheme, J. Algebraic Geom. 4 (1995), no. 4, 639-657. MR1339842 (97g:14003)

[26] Alyson Reeves and Mike Stillman, Smoothness of the lexicographic point, J. Algebraic Geom. 6 (1997), no. 2, 235-246. MR1489114 (98m:14003)

[27] G. Valla, On the Betti numbers of perfect ideals, Compositio Math. 91 (1994), no. 3, 305-319. MR:1273653 (95d:13012)

Department of Mathematics, University of Kentucky, 715 Patterson Office Tower, LEXINGTON, KENTUCKY 40506-0027

E-mail address: dennikm@gmail.com

Department of Mathematics, University of Kentucky, 715 Patterson Office Tower, LeXington, KentuCKY 40506-0027

E-mail address: uwe.nagel@uky.edu 